\title{
NONCOMMUTATIVE MATRIX JORDAN ALGEBRAS
}

\author{
ROBERT B. BROWN AND NORA C. HOPKINS
}

\begin{abstract}
We consider noncommutative degree two Jordan algebras $\mathscr{J}$ of two by two matrices whose off diagonal entries are from an anticommutative algebra $\mathscr{S}$. We give generators and relations for the automorphism group of $\mathscr{J}$ and determine the derivation algebra Der $\mathscr{J}$ in terms of mappings on $\mathscr{S}$. We also give an explicit construction of all $\mathscr{S}$ for which Der $\mathscr{J}$ does not kill the diagonal idempotents and give conditions for nonisomorphic $\mathscr{S}$ 's to give isomorphic $\mathcal{J}$ 's.
\end{abstract}

\section{INTRODUCTION}

All of the algebras and vector spaces under discussion will be over a field $k$, char $k \neq 2$. Note, however, that we are not assuming finite dimensionality and we are not assuming that the algebras are associative.

Suppose $\mathscr{S}$ is an anticommutative algebra having a nondegenerate symmetric bilinear form $B$ which is associative, that is,

$$
B(\alpha \beta, \gamma)=B(\alpha, \beta \gamma)
$$

for all $\alpha, \beta, \gamma \in \mathscr{S}$. Suppose further that $s, t \in k$ with $s t \neq 0$. We define a new algebra $\mathscr{J}=\mathscr{J}(\mathscr{S}, B, s, t)$ as

$$
\mathscr{J}:=\left\{\left(\begin{array}{ll}
a & \alpha \\
\beta & b
\end{array}\right): a, b \in k, \alpha, \beta \in \mathscr{S}\right\}
$$

with addition and scalar multiplication defined componentwise and multiplication defined by

$$
\left(\begin{array}{ll}
a & \alpha \\
\beta & b
\end{array}\right)\left(\begin{array}{ll}
c & \gamma \\
\delta & d
\end{array}\right):=\left(\begin{array}{cc}
a c+B(\alpha, \delta) & a \gamma+d \alpha+t \beta \delta \\
c \beta+b \delta+s \alpha \gamma & B(\beta, \gamma)+b d
\end{array}\right)
$$

It is easy to check that with these operations $\mathcal{J}$ is a noncommutative Jordan algebra, that is, for all $x, y \in \mathcal{J}$

$$
\begin{aligned}
& x(y x)=(x y) x, \\
& x^{2}(y x)=\left(x^{2} y\right) x .
\end{aligned}
$$

These algebras were introduced in [4] as a generalization of the Zorn matrix construction of the octonions $O$.

Received by the editors December 15, 1989 and, in revised form, July 31, 1990.

1980 Mathematics Subject Classification (1985 Revision). Primary 17A15, 17A36; Secondary $17 \mathrm{~B} 60$.

Key words and phrases. Degree two noncommutative Jordan algebra, derivation algebra, automorphism group, isomorphism. 
Example 1.5. Take $\mathscr{S}=k^{3}$, three-dimensional vectors over $k$, with the vector cross product as the algebra multiplication. Define $B$ on $\mathscr{S}$ by $B(\alpha, \beta)=$ $-\alpha \cdot \beta$, where $\alpha \cdot \beta$ denotes the dot product of $\alpha$ and $\beta$. Then $\mathscr{J}=$ $\mathscr{J}(\mathscr{S}, B, 1,1)$ is the Zorn matrix construction of the split octonions $O$ (see [2]).

These generalizations of $O$ were futher studied in [5]. Note that the Zorn matrix realization of $O$ in Example 1.5 makes the computation of $G_{2}$ as Der $O$ easy (see [2]), where the derivation algebra Der $\mathscr{J}$ of $\mathscr{J}$ is the Lie algebra of all $D \in$ End $_{k} \mathscr{J}$ satisfying

$$
(x y) D=(x D) y+x(y D)
$$

for all $x, y \in \mathscr{J}$. Our goal in this paper is to compute Der $\mathscr{J}$ for all $\mathscr{J}$ whose product is given by (1.2) and to also compute the automorphism group

$$
\text { Aut } \mathscr{J}:=\{A \in G L(\mathscr{J}) \mid(x y) A=(x A)(y A) \text { for all } x, y \in \mathscr{J}\}
$$

of $\mathscr{J}$.

The assumption that $B$ is nondegenerate is motivated by the following result from [4]:

Theorem 1.7. $\mathcal{J}$ is simple if and only if $B$ is nondegenerate.

Since the nondegeneracy of $B$ will be preserved by field extensions, we get the following corollary:

Corollary 1.8. $\mathcal{J}$ is central simple.

If $x=\left(\begin{array}{ll}a & \alpha \\ \beta & b\end{array}\right)$, then $x$ satisfies the quadratic equation

$$
x^{2}-(a+b) x+(a b-B(\alpha, \beta)) I=0
$$

where $I=\left(\begin{array}{ll}1 & 0 \\ 0 & 1\end{array}\right)$. Hence $\mathcal{J}$ is a degree two algebra (see [3] for the definition). We also note that if $y=\left(\begin{array}{ll}c & \gamma \\ \delta & d\end{array}\right)$, the bilinear form $C($,$) on \mathscr{J}$ defined by

$$
C(x, y):=\operatorname{trace}(x y)=a c+b d+B(\alpha, \delta)+B(\beta, \gamma)
$$

is nondegenerate, symmetric, and associative. Hence if we let $\mathscr{S}$ be $\mathscr{J}$ with the product $[x, y]:=x y-y x$ we can construct a new Jordan algebra $\mathscr{J}^{\prime}=$ $\mathscr{J}\left(\mathscr{S}, C, s^{\prime}, t^{\prime}\right)$.

The organization of the paper is as follows: In $\S 2$ we give a basic decomposition of Der $\mathscr{J}$ in terms of

$$
\mathscr{G}=\left\{D \in \operatorname{Der} \mathscr{J} \mid\left(\begin{array}{cc}
1 & 0 \\
0 & -1
\end{array}\right) D=\left(\begin{array}{ll}
0 & 0 \\
0 & 0
\end{array}\right)\right\}
$$

(Theorem 2.9) and characterize $\mathscr{G}$ in terms of mappings on $\mathscr{S}$ having certain properties $((2.1),(2.2)$, and Lemma 2.4$)$. In $\S 3$ we show that the codimension of $\mathscr{G}$ in Der $\mathscr{J}$ is 0,2 , or 6 (Theorem 3.8) and in fact, if $\operatorname{dim} \mathscr{S} \geq 4$, this codimension, codim $\mathscr{G}$, is either 0 or 2 (Theorem 3.4). We give instances in Example 3.6 of algebras $\mathscr{S}$ for which $\operatorname{dim} \mathscr{S} \geq 4$ and $\operatorname{codim} \mathscr{G}=2$. In $\S 4$ we show that if $\operatorname{dim} \mathscr{S} \geq 4$ and $\operatorname{codim} \mathscr{G}=2$, then $\mathscr{S}$ is one of the algebras constructed in Example 3.6 (Theorem 4.2) and we compute Der $\mathscr{J}$ for these algebras (Corollary 4.6). In $\S 5$ we give generators (Theorem 5.7 and Corollary 5.8) and relations (Proposition 5.9 and Proposition 5.13) for Aut $\mathscr{J}$. We explicitly calculate Aut $\mathscr{J}$ when $\mathscr{S}$ is one of the algebras constructed in 
Example 3.6 (Theorem 5.12 and Proposition 5.13). $\$ 6$ concerns conditions for two of these matrix Jordan algebras to be isomorphic and $\S 7$ concerns results on $\operatorname{Der} \mathscr{J}$ and Aut $\mathscr{J}$ when $s=0$ or $t=0$.

The second author wishes to gratefully acknowledge the support of Indiana State University Summer Research Grant 2-29237 during the course of this research and the hospitality of Ohio State University. She also wishes to thank Joe Ferrar for several helpful discussions.

\section{Derivations}

Let $\mathscr{G}$ be the set of $\left(D_{1}, D_{2}\right) \in \operatorname{End}_{k} \mathscr{S} \oplus \operatorname{End}_{k} \mathscr{S}$ satisfying

$$
\begin{aligned}
& B\left(\gamma D_{i}, \delta\right)=-B\left(\gamma, \delta D_{j}\right), \\
& (\gamma \delta) D_{i}=\left(\gamma D_{j}\right) \delta+\gamma\left(\delta D_{j}\right)
\end{aligned}
$$

for all $\gamma, \delta \in \mathscr{S}$, where $i, j=1,2$ and $i \neq j . \mathscr{G}$ is a Lie algebra with the Lie bracket given by $\left[\left(D_{1}, D_{2}\right),\left(E_{1}, E_{2}\right)\right]=\left(\left[D_{1}, E_{1}\right],\left[D_{2}, E_{2}\right]\right)$ and has a faithful representation on $\mathscr{J}=\mathscr{J}(\mathscr{S}, B, s, t)$ defined by

$$
\left(\begin{array}{ll}
a & \alpha \\
\beta & b
\end{array}\right)\left(D_{1}, D_{2}\right):=\left(\begin{array}{cc}
0 & \alpha D_{1} \\
\beta D_{2} & 0
\end{array}\right)
$$

for all $\left(\begin{array}{ll}a & \alpha \\ \beta & b\end{array}\right) \in \mathscr{J}$. The following lemma is a straightforward verification that $\left(D_{1}, D_{2}\right)$ acts as a derivation on $\mathscr{J}$.

Lemma 2.4. $\mathscr{G} \subseteq \operatorname{Der} \mathscr{J}$ where the action of $\mathscr{G}$ on $\mathscr{J}$ is given by (2.3).

For $\mathscr{S}$ and $B$ as in Example $1.5 \mathrm{sl}(k, 3) \cong \mathscr{G}$ [2], where $A \mapsto\left(A,-A^{t}\right)$, so $\operatorname{dim} \mathscr{G}=8$. Since $\operatorname{dim} \operatorname{Der} O=14$, clearly there are derivations of $O$ not in $\mathscr{G}$, i.e., $\mathscr{G} \neq \operatorname{Der} \mathscr{J}$. For $a \in k$ let $V_{a}$ be the linear subspace of all $\alpha \in \mathscr{S}$ such that for all $\gamma, \delta \in \mathscr{S}$

$$
a \alpha(\gamma \delta)=B(\alpha, \gamma) \delta-B(\alpha, \delta) \gamma .
$$

Lemma 2.6. $\alpha \in V_{a}$ if and only if for all $\gamma, \delta \in \mathscr{S}$

$$
a(\alpha \gamma) \delta=B(\gamma, \delta) \alpha-B(\alpha, \delta) \gamma .
$$

Proof. Suppose $\alpha$ satisfies (2.6.1) for all $\gamma, \delta \in \mathscr{S}$. Then for all $\gamma, \delta, \varepsilon \in \mathscr{S}$

$$
\begin{aligned}
-a B(\gamma, \alpha(\delta \varepsilon)) & =-a B(\gamma \alpha, \delta \varepsilon)=a B(\alpha \gamma, \delta \varepsilon)=a B((\alpha \gamma) \delta, \varepsilon) \\
& =B(\gamma, \delta) B(\alpha, \varepsilon)-B(\alpha, \delta) B(\gamma, \varepsilon) \\
& =-B(\gamma, B(\alpha, \delta) \varepsilon-B(\alpha, \varepsilon) \delta)
\end{aligned}
$$

since $B$ is associative and $\mathscr{S}$ is anticommutative. Hence, by the nondegeneracy of $B, a \alpha(\delta \varepsilon)=B(\alpha, \delta) \varepsilon-B(\alpha, \varepsilon) \delta$ which is (2.5). The converse is similar.

Another straightforward verification using Lemma 2.6 gives the following result.

Lemma 2.7. Suppose $\alpha, \beta \in V_{s t}$. Define $E_{\alpha, \beta} \in$ End $\mathscr{J}$ by

$$
\left(\begin{array}{ll}
c & \gamma \\
\delta & d
\end{array}\right) E_{\alpha, \beta}:=\left(\begin{array}{cc}
-B(\beta, \gamma)-B(\alpha, \delta) & (c-d) \alpha-t \beta \delta \\
(c-d) \beta+s \alpha \gamma & B(\beta, \gamma)+B(\alpha, \delta)
\end{array}\right) .
$$

Then $E_{\alpha, \beta} \in \operatorname{Der} \mathscr{J}$. 
Clearly $E_{\alpha, \beta} \notin \mathscr{G}$ if $\alpha \neq 0$ or $\beta \neq 0$ since

$$
\left(\begin{array}{cc}
1 & 0 \\
0 & -1
\end{array}\right) E_{\alpha, \beta}=2\left(\begin{array}{cc}
0 & \alpha \\
\beta & 0
\end{array}\right) \text {. }
$$

Also by (2.8) $\operatorname{dim} \mathscr{H}=2 \operatorname{dim} V_{s t}$ for $\mathscr{H}:=\left\{E_{\alpha, \beta} \mid \alpha, \beta \in V_{s t}\right\}$. For $\mathscr{S}$ and $B$ as in Example 1.5, $V_{1}=\mathscr{S}$, giving $\operatorname{dim} \mathscr{H}=6$ and so $\operatorname{Der} O=\mathscr{G} \oplus \mathscr{H}$ as a vector space. Note that $\mathscr{H}$ is not a subalgebra in this case. The same decomposition of Der $\mathscr{J}$ holds in the general case:

Theorem 2.9. Der $\mathscr{J}=\mathscr{G} \oplus \mathscr{H}$ as a vector space, where $\mathscr{H}:=\left\{E_{\alpha, \beta} \mid \alpha, \beta \in\right.$ $\left.V_{s t}\right\}$.

Proof. Lemmas 2.4 and 2.7 establish $\mathscr{H} \oplus \mathscr{H} \subseteq$ Der $\mathscr{J}$. To finish the proof we must show that if $D \in \operatorname{Der} \mathscr{J}$, then $D \in \mathscr{G} \oplus \mathscr{H}$. Therefore, suppose $D \in \operatorname{Der} \mathcal{J}$. $\left(\begin{array}{ll}1 & 0 \\ 0 & 1\end{array}\right) D=\left(\begin{array}{ll}0 & 0 \\ 0 & 0\end{array}\right)$ since $\left(\begin{array}{ll}1 & 0 \\ 0 & 1\end{array}\right)$ is the identity of $\mathscr{J}$. Since $\left(\begin{array}{cc}1 & 0 \\ 0 & -1\end{array}\right)^{2}=$ $\left(\begin{array}{ll}1 & 0 \\ 0 & 1\end{array}\right)$, if $\left(\begin{array}{cc}1 & 0 \\ 0 & -1\end{array}\right) D=\left(\begin{array}{ll}a & \alpha \\ \beta & b\end{array}\right)$, then the derivation condition $(1.6)$ gives

$$
\left(\begin{array}{ll}
0 & 0 \\
0 & 0
\end{array}\right)=\left(\begin{array}{ll}
a & \alpha \\
\beta & b
\end{array}\right)\left(\begin{array}{cc}
1 & 0 \\
0 & -1
\end{array}\right)+\left(\begin{array}{cc}
1 & 0 \\
0 & -1
\end{array}\right)\left(\begin{array}{ll}
a & \alpha \\
\beta & b
\end{array}\right),
$$

so $2 a=0$ and $-2 b=0$. Hence

$$
\left(\begin{array}{cc}
1 & 0 \\
0 & -1
\end{array}\right) D=\left(\begin{array}{cc}
0 & \alpha \\
\beta & 0
\end{array}\right)
$$

for some $\alpha, \beta \in \mathscr{S}$. Now define mappings $D_{11}, D_{22}, E_{11}, E_{22}$ from $\mathscr{S}$ to $k$ and mappings $D_{12}, D_{21}, E_{12}, E_{21}$ from $\mathscr{S}$ to $\mathscr{S}$ by

$$
\left(\begin{array}{ll}
0 & \gamma \\
0 & 0
\end{array}\right) D=\left(\begin{array}{ll}
\gamma D_{11} & \gamma D_{12} \\
\gamma D_{21} & \gamma D_{22}
\end{array}\right)
$$

and

$$
\left(\begin{array}{ll}
0 & 0 \\
\delta & 0
\end{array}\right) D=\left(\begin{array}{ll}
\delta E_{11} & \delta E_{12} \\
\delta E_{21} & \delta E_{22}
\end{array}\right)
$$

for all $\gamma, \delta \in \mathscr{S}$. We will use the derivation condition (1.6) to show $\alpha, \beta \in$ $V_{s t},\left(D_{12}, E_{21}\right) \in \mathscr{G}$, and $D=\left(D_{12}, E_{21}\right)+\frac{1}{2} E_{\alpha, \beta}$, thus showing $D \in \mathscr{G} \oplus \mathscr{H}$.

Using (1.6) and definitions (2.9.2) and (2.9.3) on $\left(\begin{array}{cc}0 & 0 \\ s \gamma \delta & 0\end{array}\right)=\left(\begin{array}{ll}0 & \gamma \\ 0 & 0\end{array}\right)\left(\begin{array}{ll}0 & \delta \\ 0 & 0\end{array}\right)$ yields for all $\gamma, \delta \in \mathscr{S}$

$$
\begin{aligned}
& s(\gamma \delta) E_{11}=B\left(\gamma, \delta D_{21}\right), \\
& s(\gamma \delta) E_{22}=B\left(\gamma D_{21}, \delta\right), \\
& s(\gamma \delta) E_{12}=\left(\gamma D_{11}\right) \delta+\left(\delta D_{22}\right) \gamma, \\
& s(\gamma \delta) E_{21}=s\left(\gamma D_{12}\right) \delta+s \gamma\left(\delta D_{12}\right) .
\end{aligned}
$$

A similar computation using $\left(\begin{array}{cc}0 & t \gamma \delta \\ 0 & 0\end{array}\right)=\left(\begin{array}{ll}0 & 0 \\ \gamma & 0\end{array}\right)\left(\begin{array}{ll}0 & 0 \\ \delta & 0\end{array}\right)$ yields for all $\gamma, \delta \in \mathscr{S}$

$$
\begin{aligned}
& t(\gamma \delta) D_{11}=B\left(\gamma E_{12}, \delta\right), \\
& t(\gamma \delta) D_{22}=B\left(\gamma, \delta E_{12}\right), \\
& t(\gamma \delta) D_{21}=\left(\gamma E_{22}\right) \delta+\left(\delta E_{11}\right) \gamma, \\
& t(\gamma \delta) D_{12}=t\left(\gamma E_{21}\right) \delta+t \gamma\left(\delta E_{21}\right) .
\end{aligned}
$$


Now since $\left(\begin{array}{ll}0 & \gamma \\ 0 & 0\end{array}\right)=\left(\begin{array}{cc}1 & 0 \\ 0 & -1\end{array}\right)\left(\begin{array}{ll}0 & \gamma \\ 0 & 0\end{array}\right)$, we see by $(1.6)$ that

$$
\begin{aligned}
& \gamma D_{21}=\frac{1}{2} s \alpha \gamma, \\
& \gamma D_{22}=\frac{1}{2} B(\beta, \gamma),
\end{aligned}
$$

and since $\left(\begin{array}{cc}0 & -\gamma \\ 0 & 0\end{array}\right)=\left(\begin{array}{ll}0 & \gamma \\ 0 & 0\end{array}\right)\left(\begin{array}{cc}1 & 0 \\ 0 & -1\end{array}\right)$, we see that

$$
\gamma D_{11}=-\frac{1}{2} B(\beta, \gamma) \text {. }
$$

Similarly $\left(\begin{array}{cc}1 & 0 \\ 0 & -1\end{array}\right)\left(\begin{array}{ll}0 & 0 \\ \gamma & 0\end{array}\right)=\left(\begin{array}{cc}0 & 0 \\ -\gamma & 0\end{array}\right)$ and $\left(\begin{array}{ll}0 & 0 \\ \gamma & 0\end{array}\right)\left(\begin{array}{cc}1 & 0 \\ 0 & -1\end{array}\right)=\left(\begin{array}{ll}0 & 0 \\ \gamma & 0\end{array}\right)$ give

$$
\begin{aligned}
& \gamma E_{11}=-\frac{1}{2} B(\alpha, \gamma), \\
& \gamma E_{12}=-\frac{1}{2} t \beta \gamma, \\
& \gamma E_{22}=\frac{1}{2} B(\alpha, \gamma) .
\end{aligned}
$$

\section{Hence}

and

$$
\left(\begin{array}{ll}
0 & \gamma \\
0 & 0
\end{array}\right) D=\left(\begin{array}{cc}
-\frac{1}{2} B(\beta, \gamma) & \gamma D_{12} \\
\frac{1}{2} s \alpha \gamma & \frac{1}{2} B(\beta, \gamma)
\end{array}\right)
$$

$$
\left(\begin{array}{ll}
0 & 0 \\
\delta & 0
\end{array}\right) D=\left(\begin{array}{cc}
-\frac{1}{2} B(\alpha, \delta) & -\frac{1}{2} t \beta \delta \\
\delta E_{21} & \frac{1}{2} B(\alpha, \delta)
\end{array}\right)
$$

Thus, since

$$
\left(\begin{array}{ll}
0 & \gamma \\
0 & 0
\end{array}\right)\left(\begin{array}{ll}
0 & 0 \\
\delta & 0
\end{array}\right)=\frac{1}{2} B(\gamma, \delta)\left[\left(\begin{array}{ll}
1 & 0 \\
0 & 1
\end{array}\right)+\left(\begin{array}{cc}
1 & 0 \\
0 & -1
\end{array}\right)\right],
$$

we get

$$
B\left(\gamma D_{12}, \delta\right)+B\left(\gamma, \delta E_{21}\right)=0,
$$

and (2.6.1) for $\alpha$ and $\beta$ with $a=s t$. Hence $\alpha, \beta \in V_{s t}$. Also (2.9.7) and (2.9.11) show that $\left(D_{12}, E_{21}\right)$ satisfies $(2.2)$, and (2.9.18) is $(2.1)$ for $\left(D_{12}, E_{21}\right)$ so $\left(D_{12}, E_{21}\right) \in \mathscr{G}$.

Finally, let $\widetilde{D}=D-\left(D_{12}, E_{21}\right) \in \operatorname{Der} \mathscr{J}$, i.e.,

$$
\begin{gathered}
\left(\begin{array}{ll}
1 & 0 \\
0 & 1
\end{array}\right) \widetilde{D}=\left(\begin{array}{ll}
0 & 0 \\
0 & 0
\end{array}\right), \quad\left(\begin{array}{cc}
1 & 0 \\
0 & -1
\end{array}\right) \widetilde{D}=\left(\begin{array}{ll}
0 & \alpha \\
\beta & 0
\end{array}\right), \\
\left(\begin{array}{ll}
0 & \gamma \\
0 & 0
\end{array}\right) \widetilde{D}=\left(\begin{array}{cc}
-\frac{1}{2} B(\beta, \gamma) & 0 \\
\frac{1}{2} s \alpha \gamma & \frac{1}{2} B(\beta, \gamma)
\end{array}\right),
\end{gathered}
$$

and

$$
\left(\begin{array}{ll}
0 & 0 \\
\delta & 0
\end{array}\right) \widetilde{D}=\left(\begin{array}{cc}
-\frac{1}{2} B(\alpha, \delta) & -\frac{1}{2} t \beta \delta \\
0 & \frac{1}{2} B(\alpha, \delta)
\end{array}\right)
$$

Then

$$
\begin{aligned}
\left(\begin{array}{ll}
c & \gamma \\
\delta & d
\end{array}\right) \widetilde{D} & =\frac{1}{2}\left(\begin{array}{cc}
-B(\beta, \gamma)-B(\alpha, \delta) & (c-d) \alpha-t \beta \delta \\
(c-d) \beta+s \alpha \gamma & B(\beta, \gamma)+B(\alpha, \delta)
\end{array}\right) \\
& =\left(\begin{array}{ll}
c & \gamma \\
\delta & d
\end{array}\right) \frac{1}{2} E_{\alpha, \beta}
\end{aligned}
$$

so $\widetilde{D}=\frac{1}{2} E_{\alpha, \beta} \in \mathscr{H}$. Hence $D=\left(D_{12}, E_{21}\right)+\frac{1}{2} E_{\alpha, \beta} \in \mathscr{G} \oplus \mathscr{H}$ and we are done. 
Corollary 2.10. Der $\mathscr{J}=\mathscr{G}$ iff $V_{s t}=\{0\}$.

Corollary 2.11. If $D \in \operatorname{Der} \mathscr{J}$ and $\left(\begin{array}{cc}1 & 0 \\ 0 & -1\end{array}\right) D=\left(\begin{array}{cc}0 & \alpha \\ \beta & 0\end{array}\right)$, then $\alpha, \beta \in V_{s t}$ and $D=\frac{1}{2} E_{\alpha, \beta}+F$ for some $F \in \mathscr{G}$.

The following result is another easy verification using Theorem 2.9. Recall that $C(x, y)$ is the nondegenerate symmetric bilinear form on $\mathscr{J}$ defined by (1.9).

Proposition 2.12. Let $\mathscr{L}_{C}=\{D \in$ End $\mathscr{J} \mid C(x D, y)=-C(x, y D)$ for all $x, y \in \mathscr{J}\}$. Then $\operatorname{Der} \mathscr{J} \subseteq \mathscr{L}_{C}$.

\section{Dimension of $V_{s t}$}

Lemma 3.1. Suppose $\operatorname{dim} \mathscr{S}>1$ and $V_{a} \neq\{0\}$. Then $a \neq 0$ and $V_{b}=\{0\}$ for all $b \neq a$.

Proof. Suppose $\alpha \in V_{a}$ with $\alpha \neq 0$. Then, by Lemma 2.6, $a(\alpha \gamma) \delta=B(\gamma, \delta) \alpha-$ $B(\alpha, \delta) \gamma$ for all $\gamma, \delta \in \mathscr{S}$. If $a=0$, then $B(\gamma, \delta) \alpha=B(\alpha, \delta) \gamma$ for all $\gamma, \delta \in \mathscr{S}$, which is impossible since $\operatorname{dim} \mathscr{S}>1$. Now suppose $\beta \in V_{b}$ where $b \neq a$. So, by Lemma 2.6, $b(\beta \gamma) \delta=B(\gamma, \delta) \beta-B(\beta, \delta) \gamma$ for all $\gamma, \delta \in \mathscr{S}$. Hence using (2.5) for $\alpha$ yields

$$
\begin{aligned}
& b B(\alpha, \beta) \delta-b B(\alpha, \delta) \beta \\
& \quad=a b \alpha(\beta \delta)=-a b(\beta \delta) \alpha=-a B(\delta, \alpha) \beta+a B(\beta, \alpha) \delta,
\end{aligned}
$$

so $(b-a) B(\alpha, \beta) \delta=(b-a) B(\alpha, \delta) \beta$ for all $\delta \in \mathscr{S}$. Since $b \neq a$, we get $B(\alpha, \beta) \delta=B(\alpha, \delta) \beta$ for all $\delta \in \mathscr{S}$. If $\beta \neq 0$, choosing $\delta$ linearly independent of $\beta$ gives $B(\alpha, \beta)=0=B(\alpha, \delta)$, so $B(\alpha, \delta)=0$ for all $\delta \in \mathscr{S}$, contradicting the nondegeneracy of $B$. Thus $\beta=0$ and $V_{b}=\{0\}$.

Remark 3.2. Lemma 3.1 explains why $\mathscr{J}_{1}=\mathscr{J}(\mathscr{S}, B, 1,1)$ is not isomorphic to $\mathscr{J}_{2}=\mathscr{J}(\mathscr{S}, B, 1,-1)$ for $\mathscr{S}$ and $B$ as in Example 1.5, as noted in [4], since $\operatorname{Der} \mathscr{J}_{1}=\mathscr{G} \oplus \mathscr{H}$ with $\operatorname{dim} \mathscr{H}=6$ and $\operatorname{Der} \mathscr{J}_{2}=\mathscr{G}$ by Theorem 2.9, Corollary 2.10, and Lemma 3.1.

Lemma 3.3. $V_{a}$ is a subalgebra of $\mathscr{S}$.

Proof. If $\operatorname{dim} \mathscr{S}=1$ or $V_{a}=\{0\}$, the result is obvious, so suppose $\operatorname{dim} \mathscr{S}>1$ and $V_{a} \neq\{0\}$. By Lemma 3.1, $a \neq 0$. Choose $s, t \in k$ with $s t=a \neq 0$. Then if $\alpha, \beta \in V_{a}$, we have $E_{\alpha, \beta}, E_{\beta, \alpha} \in \operatorname{Der} \mathscr{J}, \mathscr{J}=\mathscr{J}(\mathscr{S}, B, s, t)$, and hence $\left[E_{\alpha, \beta}, E_{\beta, \alpha}\right] \in \operatorname{Der} \mathcal{J}$. It is easy to check that $\left(\begin{array}{cc}1 & 0 \\ 0 & -1\end{array}\right)\left[E_{\alpha, \beta}, E_{\beta, \alpha}\right]=$ $2\left(\begin{array}{cc}0 & 2 t \beta \alpha \\ 2 s \beta \alpha & 0\end{array}\right)$, so, by Corollary $2.11, \beta \alpha \in V_{s t}=V_{a}$. Hence $V_{a}$ is a subalgebra.

Theorem 3.4. If $\operatorname{dim} \mathscr{S} \geq 4$, then $\operatorname{dim} V_{a} \leq 1$.

Proof. By Lemma 3.3 if $\alpha, \beta \in V_{a}, \alpha \beta \in V_{a}$, so by (2.5)

$$
a(\alpha \beta)(\gamma \delta)=B(\alpha \beta, \gamma) \delta-B(\alpha \beta, \delta) \gamma
$$

for all $\gamma, \delta \in \mathscr{S}$. Since $\alpha \in V_{a}$, Lemma 2.6 give $a(\alpha \beta)(\gamma \delta)=B(\beta, \gamma \delta) \alpha-$ $B(\alpha, \gamma \delta) \beta$ so this and (3.4.1) give

$$
B(\alpha \beta, \gamma) \delta-B(\alpha \beta, \delta) \gamma=B(\beta, \gamma \delta) \alpha-B(\alpha, \gamma \delta) \beta
$$

for all $\alpha, \beta \in V_{a}$ and all $\gamma, \delta \in \mathscr{S}$. 
Now suppose by way of contradiction that $\alpha, \beta \in V_{a}$ are linearly independent. Since $\operatorname{dim} \mathscr{S} \geq 4$, there are $\gamma, \delta \in \mathscr{S}$ so that $\{\alpha, \beta, \gamma, \delta\}$ is linearly independent. Hence $B(\alpha \beta, \gamma)=B(\alpha \beta, \delta)=0$ and since $B(\alpha \beta, \alpha)=$ $B\left(\beta, \alpha^{2}\right)=0=B\left(\alpha, \beta^{2}\right)=B(\alpha \beta, \beta)$, we see $\alpha \beta=0$ by the nondegeneracy of $B$ and so (3.4.2) becomes $0=B(\beta, \gamma \delta) \alpha-B(\alpha, \gamma \delta) \beta$ for all $\gamma, \delta \in \mathscr{S}$. Since $\alpha$ and $\beta$ are linearly independent, we get $B(\alpha, \gamma \delta)=B(\alpha \gamma, \delta)=0$ for all $\gamma, \delta \in \mathscr{S}$, so again using the nondegeneracy of $B$ gives $\alpha \gamma=0$ for all $\gamma \in \mathscr{S}$. But then $\alpha(\gamma \delta)=0$ for all $\gamma, \delta \in \mathscr{S}$, so by $(2.5) 0=B(\alpha, \gamma) \delta-B(\alpha, \delta) \gamma$ and choosing $\delta$ linearly independent of $\gamma$ shows $B(\alpha, \gamma)=0$ for all $\gamma \in \mathscr{S}$. Thus $\alpha=0$, contradicting the choice of $\alpha$ and $\beta$. Therefore, $\operatorname{dim} V_{a} \leq 1$.

Remark 3.5. If $\operatorname{dim} \mathscr{S} \geq 4$, Theorem 3.4 implies Lemma 3.3 , and in fact Theorem 3.4 can be proven without using Lemma 3.3, as follows: If $\alpha \neq 0$ is in $V_{a}$, let $\alpha^{\perp}:=\{\gamma \mid B(\alpha, \gamma)=0\}$. If $\gamma \in \alpha^{\perp}$ and $\delta \in \mathscr{S}$ with $B(\alpha, \delta) \neq 0$, then, by (2.5) $a \alpha(\gamma \delta)=-B(\alpha, \delta) \gamma$. This, and the fact that $B(\alpha, \alpha \sigma)=B\left(\alpha^{2}, \sigma\right)=$ 0 for all $\sigma \in \mathscr{S}$, proves that $\alpha^{\perp}=\{\alpha \sigma \mid \sigma \in \mathscr{S}\}$.

Now suppose by way of contradiction that $\operatorname{dim} V_{a}>1$ and choose $\beta \in$ $V_{a}, \beta \neq 0$, with $B(\alpha, \beta)=0$. Then $\alpha \in \beta^{\perp}$ and $\alpha=\beta \sigma$ for some $\sigma \in \mathscr{S}$. If $\gamma$ is such that $B(\beta, \gamma)=B(\sigma, \gamma)=0$ then, by Lemma 2.6, $a \alpha \gamma=a(\beta \sigma) \gamma=$ $B(\sigma, \gamma) \beta-B(\beta, \gamma) \sigma=0$, so $\operatorname{codim}\{\gamma \mid \alpha \gamma=0\} \leq 2$ (where codim is the codimension). Hence $\operatorname{dim} \alpha^{\perp} \leq 2$ and so $\operatorname{dim} \mathscr{S} \leq \overline{3}$.

The next example shows that there are numerous $(\mathscr{S}, B)$ 's for which $\operatorname{dim} \mathscr{S}$ $\geq 4$ and $\operatorname{dim} V_{a}=1$.

Example 3.6. Let $W$ be a vector space of dimension at least three and having a nondegenerate symmetric bilinear form $B$ for which there is an $A \in G L(W)$ such that $A^{2}=c I$ for some nonzero scalar $c$ and $B(\gamma A, \delta)=-B(\gamma, \delta A)$ for all $\gamma, \delta \in W$. Note that the existence of such an $A$ forces $\operatorname{dim} W$ to be even if $\operatorname{dim} W<\infty$, since $\langle\gamma, \delta\rangle:=B(\gamma A, \delta)$ is a nondegenerate skew symmetric bilinear form on $W$, and nondegenerate skew symmetric forms only exist on even dimensional spaces.

Let $\mathscr{S}=k \alpha \oplus W$, fix $d \in k, d \neq 0$, and extend $B$ to $\mathscr{S}$ by

$$
B(a \alpha+\gamma, b \alpha+\delta):=a b c d+B(\gamma, \delta)
$$

for $\gamma, \delta \in W$. Define a multiplicaton on $\mathscr{S}$ by

$$
(a \alpha+\gamma)(b \alpha+\delta):=B(\alpha, \alpha)^{-1} B(\gamma A, \delta) \alpha+(a \delta-b \gamma) A
$$

for $\gamma, \delta \in W$. With this multiplication $\mathscr{S}$ is an anticommutative algebra, $B$ is associative, and $k \alpha=V_{d}$.

Finally we consider the possibilities when $\operatorname{dim} \mathscr{S} \leq 3$.

Proposition 3.7. (i) If $\operatorname{dim} \mathscr{S}=1$, then $\mathscr{S}$ is abelian and $\mathscr{S}=V_{1}$, so $\operatorname{dim} V_{1}=$ 1.

(ii) If $\operatorname{dim} \mathscr{S}=2$, then $\mathscr{S}$ is abelian and $V_{a}=\{0\}$ for all $a \in k$.

(iii) If $\operatorname{dim} \mathscr{S}=3$, then either $\mathscr{S}$ is abelian and $V_{a}=\{0\}$ for all $a \in k$, or $\mathscr{S}$ is simple and $\mathscr{S}=V_{d}$ for some $d \in k$, in which case $\mathscr{S}$ is a Lie algebra of type $A_{1}$ and $B$ is a multiple of the Killing form. Thus $\operatorname{dim} V_{a}=0$ or 3 .

Proof. Since $\mathscr{S}$ is anticommutative, $\alpha^{2}=0$ for all $\alpha \in \mathscr{S}$. If $\alpha, \beta \in \mathscr{S}$, then $B(\alpha \beta, \alpha)=B\left(\beta, \alpha^{2}\right)=0=B\left(\alpha, \beta^{2}\right)=B(\alpha \beta, \beta)$ since $B$ is associative, so $\alpha \beta$ is orthogonal to both $\alpha$ and $\beta$ relative to $B$. This shows that $\mathscr{S}$ is 
abelian if $\operatorname{dim} \mathscr{S}=1$ or $\operatorname{dim} \mathscr{S}=2$. Then if $\operatorname{dim} \mathscr{S}=1,(2.5)$ is trivially satisfied with $a=1$, so $\mathscr{S}=V_{1}$.

If $\mathscr{S}$ is abelian and $\operatorname{dim} \mathscr{S}>1$, then $(2.5)$ gives $B(\alpha, \gamma) \delta=B(\alpha, \delta) \gamma$ for all $\alpha \in V_{a}, \gamma, \delta \in \mathscr{S}$, so choosing $\gamma$ and $\delta$ linearly independent of each other gives $B(\alpha, \gamma)=0$ for all $\gamma \in \mathscr{S}$. Thus $\alpha=0$ and $V_{a}=\{0\}$.

This only leaves the case $\operatorname{dim} \mathscr{S}=3, \mathscr{S}$ not abelian, to be considered. Let $\{\alpha, \beta, \gamma\}$ be an orthogonal basis of $\mathscr{S}$. Then $\alpha \beta=a \gamma, \beta \gamma=b \alpha, \gamma \alpha=c \beta$ for some $a, b, c \in k$. Now using the associativity of $B$ gives $a B(\gamma, \gamma)=$ $B(\alpha \beta, \gamma)=B(\alpha, \beta \gamma)=b B(\alpha, \alpha)=B(\beta, \gamma \alpha)=c B(\beta, \beta)$. A straightforward verification shows that $\mathscr{S}=V_{d}$ with $d=-B(\alpha, \alpha) a^{-1} c^{-1}$. But then by (2.5) $\mathscr{S}$ satisfies the Jacobi identity and hence must be a Lie algebra of type $A_{1}$ since $\mathscr{S}$ is not solvable. Since $B$ is associative and $\mathscr{S}$ is simple, $B$ must be a multiple of the Killing form.

Theorem 3.8. For all $a \in k \operatorname{dim} V_{a}=0,1$, or 3 , and $\operatorname{dim} V_{a}=3$ for some $a \in k$ iff $\mathscr{S}$ is a Lie algebra of type $A_{1}$.

Proof. This follows from Theorem 3.4 and Proposition 3.7.

\section{The $\operatorname{dim} V_{s t}=1$ CASE}

In this section we will show that if $\operatorname{dim} \mathscr{S} \geq 4$ and $\operatorname{dim} V_{a}=1$ for some $a \in k$, then $\mathscr{S}$ is one of the algebras constructed in Example 3.6. We will also compute $\operatorname{Der} \mathscr{J}(\mathscr{S}, B, s, t)$ in this case.

Lemma 4.1. Suppose $\operatorname{dim} \mathscr{S} \geq 4$ and $\operatorname{dim} V_{a}=1$. Then $\mathscr{S}$ is simple.

Proof. Suppose $I \triangleleft \mathscr{S}$ with $I \neq\{0\}$ and suppose $0 \neq \gamma \in I, 0 \neq \alpha \in V_{a}$, and $\delta \in \mathscr{S}$ with $B(\gamma, \delta) \neq 0$. Then $(\alpha \gamma) \delta \in I$ and $B(\alpha, \delta) \gamma \in I$, so by Lemma $2.6 B(\gamma, \delta) \alpha \in I$, giving $\alpha \in I$. Now choose $\delta \in \mathscr{S}$ with $B(\alpha, \delta) \neq 0$. Then for all $\eta \in \mathscr{S}, a(\alpha \eta) \delta-B(\eta, \delta) \alpha=-B(\alpha, \delta) \eta \in I$, again by Lemma 2.6 , so $\eta \in I$ and $I=\mathscr{S}$.

Theorem 4.2. Suppose $\operatorname{dim} \mathscr{S} \geq 4$ and $\operatorname{dim} V_{a}=1$. Then $\mathscr{S}$ is the algebra constructed in Example 3.6. Hence if $\operatorname{dim} \mathscr{S}<\infty, \operatorname{dim} \mathscr{S}$ is odd.

Proof. Suppose $V_{a}=k \alpha$. First suppose $B(\alpha, \alpha)=0$. Then there is a $\beta \in \mathscr{S}$ with $B(\alpha, \beta)=1=B(\beta, \alpha)$ and $B(\beta, \beta)=0$. We can decompose $\mathscr{S}$ relative to $B$ as $\mathscr{S}=(k \alpha \oplus k \beta) \perp W$. Then for $\gamma, \delta \in W$ we get the following from (2.5) and (2.6.1):

$$
\begin{aligned}
(\alpha \gamma) \delta & =a^{-1} B(\gamma, \delta) \alpha, \\
\alpha(\alpha \beta) & =-a^{-1} \alpha, \\
\beta(\alpha \gamma) & =a^{-1} \gamma .
\end{aligned}
$$

Now suppose $\alpha \beta=c \alpha+d \beta+\eta$ for some $\eta \in W$. Then $c=B(\alpha \beta, \beta)=$ $B\left(\alpha, \beta^{2}\right)=0$ and $d=B(\alpha \beta, \alpha)=B\left(\beta, \alpha^{2}\right)=0$, so $\alpha \beta \in W$. By (4.2.2) and (4.2.1) for $\delta \in W$

$$
\alpha \delta=-a[\alpha(\alpha \beta)] \delta=-a a^{-1} B(\alpha \beta, \delta) \alpha=-B(\alpha \beta, \delta) \alpha,
$$

so by (4.2.3) $\beta(\alpha \delta)=a^{-1} \delta=\beta[-B(\alpha \beta, \delta) \alpha]=-B(\alpha \beta, \delta) \beta \alpha$. Thus $\operatorname{dim} W$ $=1$ and $\operatorname{dim} \mathscr{S}=3$, contradicting the hypothesis $\operatorname{dim} \mathscr{S} \geq 4$. Hence no such $\mathscr{S}$ exists. 
Thus $B(\alpha, \alpha) \neq 0$. Again we decompose $\mathscr{S}$ relative to $B$ as $\mathscr{S}=k \alpha \perp W$ and get the following from (2.5) and (2.6.1) for $\gamma, \delta \in W$ :

$$
\begin{aligned}
\alpha(\gamma \delta) & =0, \\
(\alpha \gamma) \delta & =a^{-1} B(\gamma, \delta) \alpha, \\
\alpha(\alpha \gamma) & =a^{-1} B(\alpha, \alpha) \gamma .
\end{aligned}
$$

Now suppose $\gamma, \delta \in W$ and $\gamma \delta=b \alpha+\eta$ for some $\eta \in W$. Then by (4.2.4), $0=\alpha(\gamma \delta)=\alpha(b \alpha+\eta)=\alpha \eta$. Hence using (4.2.6) gives $0=\alpha 0=\alpha(\alpha \eta)=$ $a^{-1} B(\alpha, \alpha) \eta$, so $\eta=0$ and $\gamma \delta=b \alpha$ for some $b \in k$. In fact, $b B(\alpha, \alpha)=$ $B(\gamma \delta, \alpha)=B(\delta, \alpha \gamma)$, so $b=B(\alpha, \alpha)^{-1} B(\alpha \gamma, \delta)$. Thus for all $\gamma, \delta \in W$

$$
\gamma \delta=B(\alpha, \alpha)^{-1} B(\alpha \gamma, \delta) \alpha .
$$

If $\gamma \in W$ and $\alpha \gamma=c \alpha+\eta$ for some $\eta \in W$, by (4.2.5)

$$
\begin{aligned}
a^{-1} B(\gamma, \gamma) \alpha & =(\alpha \gamma) \gamma=(c \alpha+\eta) \gamma=c \alpha \gamma+\eta \gamma \\
& =c(c \alpha+\eta)+B(\alpha, \alpha)^{-1} B(\alpha \eta, \gamma) \alpha \\
& =\left[c^{2}+B(\alpha, \alpha)^{-1} B(\alpha \eta, \gamma)\right] \alpha+c \eta
\end{aligned}
$$

by (4.2.7), so $a^{-1} B(\gamma, \gamma)=c^{2}+B(\alpha, \alpha)^{-1} B(\alpha \eta, \gamma)$ and $c \eta=0$. Hence either $c=0$ or $\eta=0$. But if $\eta=0, \alpha \gamma=c \alpha$, so by (4.2.6) $\gamma=a B(\alpha, \alpha)^{-1} \alpha(\alpha \gamma)=$ $a B(\alpha, \alpha)^{-1} c \alpha^{2}=0$. Thus $\eta \neq 0$ so $c=0$ and $\alpha \gamma=\eta \in W$ and $B(\alpha \eta, \gamma)=$ $a^{-1} B(\alpha, \alpha) B(\gamma, \gamma)$. Hence for $\gamma \in W$,

(4.2.8) $\alpha \gamma=\eta$ for some $\eta \in W$, where $B(\alpha \gamma, \eta)=-a^{-1} B(\alpha, \alpha) B(\gamma, \gamma)$.

Note that since $\eta=\alpha \gamma, B(\alpha \gamma, \alpha \gamma)=-a^{-1} B(\alpha, \alpha) B(\gamma, \gamma)$.

$\mathscr{S}$ is simple by Lemma 4.1, so $W=\alpha W$ since $W^{2} \subseteq k \alpha$ by (4.2.7). Let $A$ : $W \rightarrow W$ be defined by $\gamma A=\alpha \gamma$, so we have $B(\gamma A, \gamma A)=-a^{-1} B(\alpha, \alpha) B(\gamma, \gamma)$ and $A^{2}=a^{-1} B(\alpha, \alpha) I$ by (4.2.6), and we get from (4.2.7) and (4.2.8) that

$$
(c \alpha+\gamma)(d \alpha+\delta)=B(\alpha, \alpha)^{-1} B(\gamma A, \delta) \alpha+(c \delta-d \gamma) A
$$

which is (3.6.1). As noted in Example 3.6, $\operatorname{dim} W$ is even if $\operatorname{dim} W<\infty$, so $\operatorname{dim} \mathscr{S}$ is odd.

We need two lemmas for the computation of $\operatorname{Der} \mathscr{J}(\mathscr{S}, B, s, t)$.

Lemma 4.3. If $\alpha \in V_{a}$ and $\left(D_{1}, D_{2}\right) \in \mathscr{G}$, then $\alpha D_{1}, \alpha D_{2} \in V_{a}$.

Proof. This is a straightforward verificaton done by applying $D_{1}$ and $D_{2}$ to (2.5).

Lemma 4.4. Suppose $\operatorname{dim} \mathscr{S} \geq 4$ and $\operatorname{dim} V_{a}=1$. Define $\pi: \mathscr{G} \rightarrow$ End $\mathscr{S}$ by $\left(D_{1}, D_{2}\right) \pi:=D_{1}$. Then $\mathscr{G} \cong \mathscr{G} \pi \cong \mathscr{L} \oplus k c$, where $\mathscr{L}$ is a symplectic Lie algebra with $\alpha l=0$ for all $l \in \mathscr{L}, \alpha \in V_{a}$, and $c$ is a central element of $\mathscr{G}$ defined by

$$
(b \alpha+\gamma) c:=b \alpha-\frac{1}{2} \gamma
$$

for all $\gamma \in \mathscr{S}$ such that $B(\alpha, \gamma)=0, \alpha \in V_{a}$.

Proof. By Theorem 4.2 if $V_{a}=k \alpha$, then $\mathscr{S}=k \alpha \perp W$ relative to $B$ and the multiplication in $\mathscr{S}$ is given by (4.2.9), where $A \in G L(W)$ is defined by $\gamma A:=$ $\alpha \gamma$ and satisfies $A^{2}=a^{-1} B(\alpha, \alpha) I$ and $B(\gamma A, \gamma A)=-a^{-1} B(\alpha, \alpha) B(\gamma, \gamma)$ for all $\gamma \in W$. Then $\langle\gamma, \delta\rangle:=B(\gamma A, \delta)$ is a nondegenerate skew-symmetric 
bilinear form on $W$. Let $\mathscr{L}:=\{D \in$ End $W \mid\langle\gamma D, \delta\rangle=-\langle\gamma, \delta D\rangle$ for all $\gamma, \delta \in W\}$ and define $\bar{D} \in$ End $\mathscr{S}$ for $D \in$ End $W$ by $(a \alpha+\gamma) \bar{D}:=\gamma D$ for all $\gamma \in W$. Then if $D \in \mathscr{L}$, it is easy to check that $\left(\bar{D}, \overline{A^{-1} D A}\right) \in \mathscr{G}$ and $D \mapsto\left(\bar{D}, \overline{A^{-1} D A}\right)$ is a homomorphism of Lie algebras. Also $(c,-c) \in \mathscr{G}$ so $\mathscr{L} \oplus k c$ is isomorphic to a subalgebra of $\mathscr{G}$.

Conversely, suppose $\left(D_{1}, D_{2}\right) \in \mathscr{G}$. By Lemma 4.3, $\alpha D_{i}=a_{i} \alpha$ for some $a_{i} \in k$. Since $B\left(\alpha D_{i}, \alpha\right)=-B\left(\alpha, \alpha D_{j}\right)$ we see $a_{1}=-a_{2}$. Moreover, if $\gamma \in W$ and $\gamma D_{i}=b_{i} \alpha+\gamma_{i}$, then $B\left(\gamma D_{i}, \alpha\right)=-B\left(\gamma, \alpha D_{j}\right)$ implies $b_{i}=0$, so $W D_{i} \subseteq W$. Let $\left(E_{1}, E_{2}\right)=\left(D_{1}, D_{2}\right)-a_{1}(c,-c)$, so $\left(E_{1}, E_{2}\right) \in \mathscr{G}$ and $\alpha E_{i}=0$ for $i=1,2$. Then for $\gamma \in W,(\alpha \gamma) E_{i}=\left(\alpha E_{j}\right) \gamma+\alpha\left(\gamma E_{j}\right)=\alpha\left(\gamma E_{j}\right)$ implies $\gamma A E_{i}=\gamma E_{j} A$, i.e., $\left.E_{2}\right|_{W}=A^{-1} D A$ for $D:=\left.E_{1}\right|_{W}$, so $\left(E_{1}, E_{2}\right)=$ $\left(\bar{D}, \overline{A^{-1} D A}\right)$. Also for $\gamma, \delta \in W, 0=B(\alpha, \alpha)^{-1} B(\gamma A, \delta) \alpha E_{2}=(\gamma \delta) E_{2}=$ $\left(\gamma E_{1}\right) \delta+\gamma\left(\delta E_{1}\right)=B(\alpha, \alpha)^{-1}\left[B\left(\gamma E_{1} A, \delta\right)+B\left(\gamma A, \delta E_{1}\right)\right] \alpha$, so $D \in \mathscr{L}$ and we are done.

Theorem 4.5. Suppose $\operatorname{dim} \mathscr{S} \geq 4, \operatorname{dim} V_{a}=1, \mathscr{J}=\mathscr{J}(\mathscr{S}, B, s, t)$, st $=$ $a$, and $c \in$ End $\mathscr{S}$ is defined by (4.4.1). Then $[\mathscr{H} \oplus k(c,-c)] \triangleleft \operatorname{Der} \mathscr{J}$ is isomorphic to $\operatorname{sl}(2, k)$, where $\mathscr{H}=\left\{E_{\alpha, \beta} \mid \alpha, \beta \in V_{a}\right\}, E_{\alpha, \beta}$ defined by (2.7.1). Moreover, $\mathscr{L} \triangleleft \operatorname{Der} \mathscr{J}$ so Der $\mathscr{J}$ is the direct sum of an $\operatorname{sl}(2, k)$ ideal and a symplectic ideal.

Proof. A straightforward verification shows that if $\alpha_{i}, \beta_{i} \in V_{s t}$ for $i=1,2$, then $\left[E_{\alpha_{1}, \beta_{1}}, E_{\alpha_{2}, \beta_{2}}\right]=E_{\beta, \alpha}+\left(D_{1}, D_{2}\right)$, where $\beta=2 t \beta_{1} \beta_{2}, \alpha=2 s \alpha_{1} \alpha_{2}$, and $\left(D_{1}, D_{2}\right) \in \mathscr{G}$ is defined by $\gamma D_{1}:=2 B\left(\beta_{2}, \gamma\right) \alpha_{1}-s t \beta_{2}\left(\alpha_{1} \gamma\right)-2 B\left(\beta_{1}, \gamma\right) \alpha_{2}+$ st $\beta_{1}\left(\alpha_{2} \gamma\right)$ and $\gamma D_{2}:=2 B\left(\alpha_{2}, \gamma\right) \beta_{1}-s t \alpha_{2}\left(\beta_{1} \gamma\right)-2 B\left(\alpha_{1}, \gamma\right) \beta_{2}+s t \alpha_{1}\left(\beta_{2} \gamma\right)$ for all $\gamma \in \mathscr{S}$, and it is easy to check that if $\alpha, \beta \in V_{s t}$ and $\left(D_{1}, D_{2}\right) \in \mathscr{G}$, then $\left[E_{\alpha, \beta},\left(D_{1}, D_{2}\right)\right]=E_{\alpha^{\prime}, \beta^{\prime}}$, where $\alpha^{\prime}=\alpha D_{1}$ and $\beta^{\prime}=\beta D_{2}$, which are both in $V_{s t}$ by Lemma 4.3. Since $\alpha l=0$ for all $l \in \mathscr{L} \subseteq \mathscr{G}_{\pi}$ and $(c,-c)$ is a central element of $\mathscr{G}$, we see that $\mathscr{L} \triangleleft \operatorname{Der} \mathscr{J}$. Since $\operatorname{dim} V_{s t}=1, \operatorname{dim} \mathscr{H}=2$ and $\mathscr{H}$ is spanned by $\left\{E_{\alpha, 0}, E_{0, \beta}\right\}$, where $\alpha, \beta$ are nonzero elements of $V_{s t}$ with $\beta=B(\alpha, \alpha)^{-1} \alpha$. Hence $\mathscr{H} \oplus k(c,-c)$ is three dimensional and by the previous formulas $\left[E_{\alpha, 0}, E_{0, \beta}\right]=2(c,-c),\left[E_{\alpha, 0}, 2(c,-c)\right]=2 E_{\alpha, 0}$, and $\left[E_{0, \beta}, 2(c,-c)\right]=-2 E_{0, \beta}$, so $\mathscr{H} \oplus k(c,-c)$ is isomorphic to $\operatorname{sl}(2, k)$. That $\mathscr{H} \oplus k(c,-c)$ is an ideal follows from Lemma 4.4 and Theorem 2.9.

Corollary 4.6. Suppose $\operatorname{dim} \mathscr{S} \geq 4$ and $\operatorname{dim} V_{a}=1$.

(i) If $s t=a$, then $\operatorname{Der} \mathscr{J}(\mathscr{S}, B, s, t) \cong \mathscr{L} \oplus \operatorname{sl}(2, k)$, where $\mathscr{L}$ is a symplectic Lie algebra, $\mathscr{L} \triangleleft \operatorname{Der} \mathscr{J}(\mathscr{S}, B, s, t)$, and $s l(2, k) \triangleleft \operatorname{Der} \mathscr{J}(\mathscr{S}, B, s, t)$.

(ii) If $s t \neq a$, then $\operatorname{Der} \mathscr{J}(\mathscr{S}, B, s, t) \cong \mathscr{L} \oplus k c$, where $\mathscr{L}$ is a symplectic Lie algebra, $\mathscr{L} \triangleleft \operatorname{Der} \mathscr{J}(\mathscr{S}, B, s, t)$, and $c$ is a central element of $\operatorname{Der} \mathscr{J}$ acting semisimply on $\mathscr{S}$.

Proof. For (ii) use Corollary 2.10 and Lemma 3.1.

\section{AUTOMORPHISMS}

In this section we will determine the automorphism group of $\mathscr{J}(\mathscr{S}, B, s, t)$. The following proposition is a straightforward verification.

Proposition 5.1. Suppose $\mathscr{J}=\mathscr{J}(\mathscr{S}, B, s, t)$.

(i) Suppose $R=\left(R_{1}, R_{2}\right) \in G L(\mathscr{S}) \times G L(\mathscr{S})$ such that for all $\gamma, \delta \in$ $\mathscr{S}, B\left(\gamma R_{i}, \delta R_{j}\right)=B(\gamma, \delta)$ and $(\gamma \delta) R_{i}=\left(\gamma R_{j}\right)\left(\delta R_{j}\right)$ for $i, j=1,2, i \neq j$. 
Then $A_{R} \in$ Aut $\mathscr{J}$, where $A_{R}$ is defined by

$$
\left(\begin{array}{ll}
c & \gamma \\
\delta & d
\end{array}\right) A_{R}:=\left(\begin{array}{cc}
c & \gamma R_{1} \\
\delta R_{2} & d
\end{array}\right) .
$$

(ii) Suppose $S=\left(S_{1}, S_{2}\right) \in G L(\mathscr{S}) \times G L(\mathscr{S})$ such that $B\left(\gamma S_{1}, \delta S_{2}\right)=$ $B(\gamma, \delta), s(\gamma \delta) S_{1}=t\left(\gamma S_{2}\right)\left(\delta S_{2}\right)$, and $t(\gamma \delta) S_{2}=s\left(\gamma S_{1}\right)\left(\delta S_{1}\right)$ for all $\gamma, \delta \in \mathscr{S}$. Then $B_{S} \in$ Aut $\mathcal{J}$, where $B_{S}$ is defined by

$$
\left(\begin{array}{ll}
c & \gamma \\
\delta & d
\end{array}\right) B_{S}:=\left(\begin{array}{cc}
d & \delta S_{1} \\
\gamma S_{2} & c
\end{array}\right)
$$

(iii) Suppose $\alpha, \beta \in V_{\text {st }}$. Then $E_{\alpha}, F_{\beta} \in$ Aut $\mathscr{J}$, where $E_{\alpha}$ and $F_{\beta}$ are defined by

$$
\begin{aligned}
& \left(\begin{array}{ll}
c & \gamma \\
\delta & d
\end{array}\right) E_{\alpha}:=\left(\begin{array}{cc}
c-B(\alpha, \delta) & \gamma+(c-d) \alpha-B(\alpha, \delta) \alpha \\
\delta+s \alpha \gamma & d+B(\alpha, \delta)
\end{array}\right), \\
& \left(\begin{array}{ll}
c & \gamma \\
\delta & d
\end{array}\right) F_{\beta}:=\left(\begin{array}{cc}
c+B(\beta, \gamma) & \gamma+t \beta \delta \\
\delta+(d-c) \beta-B(\beta, \gamma) \beta & d-B(\beta, \gamma)
\end{array}\right) .
\end{aligned}
$$

Note that $E_{\alpha}$ is the exponential of the derivation $E_{\alpha, 0}$ and $F_{\beta}$ is the exponential of the derivation $E_{0,-\beta}$.

We will now show that Aut $\mathcal{J}$ is generated by the $A_{R}$ 's, $B_{S}$ 's, $E_{\alpha}$ 's, and $F_{\beta}$ 's. For this suppose $E \in \operatorname{Aut} \mathscr{J}$. Then $\left(\begin{array}{ll}1 & 0 \\ 0 & 1\end{array}\right) E=\left(\begin{array}{ll}1 & 0 \\ 0 & 1\end{array}\right)$ so

$$
\left(\begin{array}{ll}
c & 0 \\
0 & d
\end{array}\right) E=\left(\begin{array}{cc}
c+a(d-c) & (c-d) \alpha \\
(d-c) \beta & d+b(c-d)
\end{array}\right)
$$

for some $a, b \in k$ and some $\alpha, \beta \in \mathscr{S}$. Also, there are linear mappings $E_{11}, E_{22}, F_{11}, F_{22}: \mathscr{S} \rightarrow k$ and $E_{12}, E_{21}, F_{12}, F_{21}: \mathscr{S} \rightarrow \mathscr{S}$ such that

$$
\left(\begin{array}{ll}
0 & \gamma \\
0 & 0
\end{array}\right) E=\left(\begin{array}{ll}
\gamma E_{11} & \gamma E_{12} \\
\gamma E_{21} & \gamma E_{22}
\end{array}\right)
$$

and

$$
\left(\begin{array}{ll}
0 & 0 \\
\delta & 0
\end{array}\right) E=\left(\begin{array}{ll}
\delta F_{11} & \delta F_{12} \\
\delta F_{21} & \delta F_{22}
\end{array}\right)
$$

for all $\gamma, \delta \in \mathscr{S}$.

Lemma 5.5. (i) $a(a-1)=b(b-1)=B(\alpha, \beta)$.

(ii) $(b-a) \alpha=0=(a-b) \beta$.

(iii) $E_{22}=-E_{11}$ and $F_{22}=-F_{11}$.

(iv) If $\alpha \neq 0$ or $\beta \neq 0$, then $a=b$.

Proof. Since $\left(\begin{array}{ll}1 & 0 \\ 0 & 0\end{array}\right) E=\left(\begin{array}{cc}1-a & \alpha \\ -\beta & b\end{array}\right)$ and $\left(\begin{array}{ll}1 & 0 \\ 0 & 0\end{array}\right)\left(\begin{array}{ll}1 & 0 \\ 0 & 0\end{array}\right)=\left(\begin{array}{ll}1 & 0 \\ 0 & 0\end{array}\right)$, the computation of $\left(\begin{array}{ll}1 & 0 \\ 0 & 0\end{array}\right) E\left(\begin{array}{ll}1 & 0 \\ 0 & 0\end{array}\right) E=\left(\begin{array}{ll}1 & 0 \\ 0 & 0\end{array}\right) E$ gives (i) and (ii). For (iii), computation of

$$
\left(\begin{array}{ll}
0 & \gamma \\
0 & 0
\end{array}\right) E\left(\begin{array}{ll}
0 & \gamma \\
0 & 0
\end{array}\right) E=\left(\begin{array}{ll}
0 & 0 \\
0 & 0
\end{array}\right) E=\left(\begin{array}{ll}
0 & 0 \\
0 & 0
\end{array}\right)
$$

yields

$$
\left(\gamma E_{11}\right)^{2}+B\left(\gamma E_{12}, \gamma E_{21}\right)=0=B\left(\gamma E_{21}, \gamma E_{12}\right)+\left(\gamma E_{22}\right)^{2}
$$

and

$$
\left(\gamma E_{11}\right)\left(\gamma E_{12}\right)+\left(\gamma E_{22}\right)\left(\gamma E_{12}\right)=0=\left(\gamma E_{11}\right)\left(\gamma E_{21}\right)+\left(\gamma E_{22}\right)\left(\gamma E_{21}\right) .
$$


By (5.5.1) $\left(\gamma E_{11}\right)^{2}=\left(\gamma E_{22}\right)^{2}$, so $\gamma E_{22}=\varepsilon \gamma E_{11}$ for $\varepsilon= \pm 1$. Therefore (5.5.2) becomes $\left(\gamma E_{11}+\varepsilon \gamma E_{11}\right)\left(\gamma E_{12}\right)=0=\left(\gamma E_{11}+\varepsilon \gamma E_{11}\right)\left(\gamma E_{21}\right)$ so either $\gamma E_{11}+\varepsilon \gamma E_{11}=0$ or $\gamma E_{12}=0=\gamma E_{21}$. But if $\gamma E_{12}=0=\gamma E_{21},(5.5 .1)$ gives $\left(\gamma E_{11}\right)^{2}=0=\left(\gamma E_{22}\right)^{2}$ so $\left(\begin{array}{ll}0 & \gamma \\ 0 & 0\end{array}\right) E=\left(\begin{array}{ll}0 & 0 \\ 0 & 0\end{array}\right)$, contradicting the nonsingularity of $E$. Hence $\gamma E_{11}+\varepsilon \gamma E_{11}=0$, so $\gamma E_{22}=-\gamma E_{11}$. A similar argument with $\left(\begin{array}{ll}0 & 0 \\ \delta & 0\end{array}\right) E\left(\begin{array}{ll}0 & 0 \\ \delta & 0\end{array}\right) E=\left(\begin{array}{ll}0 & 0 \\ 0 & 0\end{array}\right)$ shows $\delta F_{22}=-\delta F_{11}$. (iv) follows directly from (ii).

Lemma 5.6. Suppose $\alpha=0=\beta$ in (5.2). Then $E=A_{R}$, where $R=\left(E_{12}, F_{21}\right)$, or $E=B_{S}$, where $S=\left(F_{12}, E_{21}\right)$.

Proof. By Lemma 5.5(i) $a$ and $b$ can have only 0 and 1 as values. If $a=0$ and $b=1$, then $\left(\begin{array}{ll}0 & 0 \\ 0 & 1\end{array}\right) E=\left(\begin{array}{ll}0 & 0 \\ 0 & 0\end{array}\right)$, and if $a=1$ and $b=0$, then $\left(\begin{array}{ll}1 & 0 \\ 0 & 0\end{array}\right) E=$ $\left(\begin{array}{ll}0 & 0 \\ 0 & 0\end{array}\right)$, either case being a contradiction of the nonsingularity of $E$. Hence $a=b=0$ or $a=b=1$.

Suppose $a=b=0$. Then $\left(\begin{array}{ll}c & 0 \\ 0 & d\end{array}\right) E=\left(\begin{array}{ll}c & 0 \\ 0 & d\end{array}\right)$ so $\left(\begin{array}{cc}1 & 0 \\ 0 & -1\end{array}\right) E\left(\begin{array}{ll}0 & \gamma \\ 0 & 0\end{array}\right) E=\left(\begin{array}{ll}0 & \gamma \\ 0 & 0\end{array}\right) E$ yields $\gamma E_{21}=0$ and $\gamma E_{11}=0$ by Lemma 5.5 (iii). Hence

$$
\left(\begin{array}{ll}
0 & \gamma \\
0 & 0
\end{array}\right) E=\left(\begin{array}{cc}
0 & \gamma E_{12} \\
0 & 0
\end{array}\right),
$$

so $E_{12}$ must be nonsingular since $E$ is nonsingular. A similar argument with $\left(\begin{array}{cc}-1 & 0 \\ 0 & 1\end{array}\right) E\left(\begin{array}{ll}0 & 0 \\ \delta & 0\end{array}\right) E=\left(\begin{array}{ll}0 & 0 \\ \delta & 0\end{array}\right) E$ yields $\delta F_{11}=0$ and $\delta F_{12}=0$, so

$$
\left(\begin{array}{ll}
0 & 0 \\
\delta & 0
\end{array}\right) E=\left(\begin{array}{cc}
0 & 0 \\
\delta F_{21} & 0
\end{array}\right)
$$

and $F_{21}$ is nonsingular. $\left(\begin{array}{ll}0 & \gamma \\ 0 & 0\end{array}\right) E\left(\begin{array}{ll}0 & 0 \\ \delta & 0\end{array}\right) E=\left(\begin{array}{cc}B(\gamma, \delta) & 0 \\ 0 & 0\end{array}\right) E$ gives $B\left(\gamma E_{12}, \delta F_{21}\right)=$ $B(\gamma, \delta)$ for all $\gamma, \delta \in \mathscr{S}$, and $\left(\begin{array}{ll}0 & \gamma \\ 0 & 0\end{array}\right) E\left(\begin{array}{ll}0 & \eta \\ 0 & 0\end{array}\right) E=\left(\begin{array}{cc}0 & 0 \\ s \gamma \eta & 0\end{array}\right) E$ and $\left(\begin{array}{ll}0 & 0 \\ \delta & 0\end{array}\right) E\left(\begin{array}{ll}0 & 0 \\ \tau & 0\end{array}\right) E=$ $\left(\begin{array}{cc}0 & t \delta \tau \\ 0 & 0\end{array}\right) E$ yield $(\gamma \eta) F_{21}=\left(\gamma E_{12}\right)\left(\eta E_{12}\right)$ and $(\delta \tau) E_{12}=\left(\delta F_{21}\right)\left(\tau F_{21}\right)$. Thus

$$
\left(\begin{array}{ll}
c & \gamma \\
\delta & d
\end{array}\right) E=\left(\begin{array}{cc}
c & \gamma E_{12} \\
\delta F_{21} & d
\end{array}\right)=\left(\begin{array}{ll}
c & \gamma \\
\delta & d
\end{array}\right) A_{R}
$$

where $R=\left(E_{12}, F_{21}\right)$.

If $a=b=1$, then $\left(\begin{array}{ll}c & 0 \\ 0 & d\end{array}\right) E=\left(\begin{array}{ll}d & 0 \\ 0 & c\end{array}\right)$ and calculations similar to the ones above show $E=B_{S}$, where $S=\left(F_{12}, E_{21}\right)$.

We now return to the general case.

Theorem 5.7. Aut $\mathcal{J}$ is generated by the $A_{R}$ 's, $B_{S}$ 's, $E_{\alpha}$ 's, and $F_{\beta}$ 's of Proposition 5.1.

Proof. By Lemma 5.6 it only remains to deal with the case $\alpha \neq 0$ or $\beta \neq 0$. Assuming $\alpha \neq 0$, we get $a=b$ by Lemma 5.5(iv) so

$$
\left(\begin{array}{ll}
c & 0 \\
0 & d
\end{array}\right) E=\left(\begin{array}{cc}
c+a(d-c) & (c-d) \alpha \\
(d-c) \beta & d+a(c-d)
\end{array}\right) .
$$

By Lemma 5.5(iii)

$$
\left(\begin{array}{ll}
0 & \gamma \\
0 & 0
\end{array}\right) E=\left(\begin{array}{cc}
\gamma E_{11} & \gamma E_{12} \\
\gamma E_{21} & -\gamma E_{11}
\end{array}\right)
$$

and

$$
\left(\begin{array}{ll}
0 & 0 \\
\delta & 0
\end{array}\right) E=\left(\begin{array}{cc}
\delta F_{11} & \delta F_{12} \\
\delta F_{21} & -\delta F_{11}
\end{array}\right)
$$


Once we have shown $\alpha, \beta \in V_{\text {st }}$, then $E_{\alpha}, F_{\beta} \in$ Aut $\mathscr{J}$, where $E_{\alpha}$ and $F_{\beta}$ are defined by (5.1.3) and (5.1.4) respectively. If $a=1$, then $\left(\begin{array}{ll}c & 0 \\ 0 & d\end{array}\right) E E_{\alpha} F_{\beta}=\left(\begin{array}{ll}d & 0 \\ 0 & c\end{array}\right)$ since $B(\alpha, \beta)=0$ by Lemma $5.5(\mathrm{i})$, so $E E_{\alpha} F_{\beta} \in$ Aut $\mathscr{J}$ is of the form $B_{S}$ by Lemma 5.6. If $a \neq 1$ and $\alpha^{\prime}=(a-1)^{-1} \alpha$, then $\left(\begin{array}{ll}c & 0 \\ 0 & d\end{array}\right) E E_{\alpha^{\prime}} F_{-\beta}=\left(\begin{array}{ll}c & 0 \\ 0 & d\end{array}\right)$ so $E E_{\alpha^{\prime}} F_{-\beta} \in$ Aut $\mathcal{J}$ is of the form $A_{R}$ by Lemma 5.6. Thus, the proof will be complete once we have shown $\alpha, \beta \in V_{s t}$.

To see that $\alpha \in V_{s t}$ we compute the diagonal terms of $\left(\begin{array}{ll}0 & \gamma \\ 0 & 0\end{array}\right) E\left(\begin{array}{ll}0 & 0 \\ \delta & 0\end{array}\right) E=$ $\left(\begin{array}{cc}B(\gamma, \delta) & 0 \\ 0 & 0\end{array}\right) E, \quad\left(\begin{array}{ll}1 & 0 \\ 0 & 0\end{array}\right) E\left(\begin{array}{ll}0 & \gamma \\ 0 & 0\end{array}\right) E=\left(\begin{array}{ll}0 & \gamma \\ 0 & 0\end{array}\right) E$, and $\left(\begin{array}{ll}0 & 0 \\ 0 & 1\end{array}\right) E\left(\begin{array}{ll}0 & 0 \\ \delta & 0\end{array}\right) E=\left(\begin{array}{ll}0 & 0 \\ \delta & 0\end{array}\right) E$ to get the following identities:

$$
\begin{gathered}
(1-a) B(\gamma, \delta)=\left(\gamma E_{11}\right)\left(\delta F_{11}\right)+B\left(\gamma E_{12}, \delta F_{21}\right), \\
a B(\gamma, \delta)=B\left(\gamma E_{21}, \delta F_{12}\right)+\left(\gamma E_{11}\right)\left(\delta F_{11}\right), \\
a \gamma E_{11}=B\left(\alpha, \gamma E_{21}\right) \\
(1-a) \gamma E_{11}=B\left(\beta, \gamma E_{12}\right) \\
(1-a) \delta F_{11}=-B\left(\alpha, \delta F_{21}\right) \\
a \delta F_{11}=-B\left(\beta, \delta F_{12}\right)
\end{gathered}
$$

Now if $a \neq 1, E_{12}$ must be nonsingular, for if $\gamma E_{12}=0$ for some $\gamma \neq 0$, then $\gamma E_{11}=0$ by $(5.7 .7)$, so $B(\gamma, \delta)=0$ for all $\delta \in \mathscr{S}$ by (5.7.4), contradicting the nondegeneracy of $B$. Similarly, if $a \neq 1, F_{21}$ is nonsingular by (5.7.8) and (5.7.4). If $a=1$, then $E_{21}$ and $F_{12}$ must both be nonsingular by (5.7.5), (5.7.6), and (5.7.9). Computing the off-diagonal terms of $\left(\begin{array}{ll}0 & \gamma \\ 0 & 0\end{array}\right) E\left(\begin{array}{ll}0 & 0 \\ \delta & 0\end{array}\right) E=$ $\left(\begin{array}{cc}B(\gamma, \delta) & 0 \\ 0 & 0\end{array}\right) E,\left(\begin{array}{ll}0 & \gamma \\ 0 & 0\end{array}\right) E\left(\begin{array}{ll}0 & 0 \\ 0 & 1\end{array}\right) E=\left(\begin{array}{ll}0 & \gamma \\ 0 & 0\end{array}\right) E$, and $\left(\begin{array}{ll}0 & 0 \\ \delta & 0\end{array}\right) E\left(\begin{array}{ll}1 & 0 \\ 0 & 0\end{array}\right) E=\left(\begin{array}{ll}0 & 0 \\ \delta & 0\end{array}\right) E$ yields the following identities:

(5.7.10) $B(\gamma, \delta) \alpha=\left(\gamma E_{11}\right)\left(\delta F_{12}\right)-\left(\delta F_{11}\right)\left(\gamma E_{12}\right)+t\left(\gamma E_{21}\right)\left(\delta F_{21}\right)$,

$(5.7 .11)-B(\gamma, \delta) \beta=\left(\delta F_{11}\right)\left(\gamma E_{21}\right)-\left(\gamma E_{11}\right)\left(\delta F_{21}\right)+s\left(\gamma E_{12}\right)\left(\delta F_{12}\right)$,

$$
\begin{gathered}
(1-a) \gamma E_{21}=-\left(\gamma E_{11}\right) \beta-s\left(\gamma E_{12}\right) \alpha, \\
a \gamma E_{12}=-\left(\gamma E_{11}\right) \alpha+t\left(\gamma E_{21}\right) \beta, \\
(1-a) \delta F_{12}=\left(\delta F_{11}\right) \alpha-t\left(\delta F_{21}\right) \beta \\
a \delta F_{21}=\left(\delta F_{11}\right) \beta+s\left(\delta F_{12}\right) \alpha .
\end{gathered}
$$

Suppose $a \neq 1$. Solving (5.7.8) for $\delta F_{11}$ and (5.7.7) for $\gamma E_{11}$ and substituting into (5.7.4) yields

(5.7.16) $(1-a) B(\gamma, \delta)=-(1-a)^{-2} B\left(\beta, \gamma E_{12}\right) B\left(\alpha, \delta F_{21}\right)+B\left(\gamma E_{12}, \delta F_{21}\right)$.

Solving for $\gamma E_{11}$ in (5.7.7), $\delta F_{11}$ in (5.7.8), $\gamma E_{21}$ in (5.7.12), and $\delta F_{12}$ in (5.7.14) and eliminating these quantities from (5.7.10) gives

$$
\begin{aligned}
B(\gamma, \delta) \alpha= & -(1-a)^{-3} B\left(\gamma E_{12}, \beta\right) B\left(\delta F_{21}, \alpha\right) \alpha \\
& +(1-a)^{-1} B\left(\alpha, \delta F_{21}\right)\left(\gamma E_{12}\right) \\
& +(1-a)^{-1} s t\left[\alpha\left(\gamma E_{12}\right)\right]\left(\delta F_{21}\right) .
\end{aligned}
$$

We now multiply (5.7.17) by $(1-a)$ and (5.7.16) by $\alpha$ to obtain

$$
0=B\left(\alpha, \delta F_{21}\right)\left(\gamma E_{12}\right)+s t\left[\alpha\left(\gamma E_{12}\right)\right]\left(\delta F_{21}\right)-B\left(\gamma E_{12}, \delta F_{21}\right) \alpha .
$$

Hence $\alpha$ satisfies (2.6.1) since $E_{12}$ and $F_{21}$ are nonsingular when $a \neq 1$, so $\alpha \in V_{s t}$. 
If $a=1$, a similar argument using (5.7.5), (5.7.6), (5.7.9), (5.7.11), (5.7.13), and (5.7.15) proves $\beta \in V_{s t}$. The computations showing $\beta \in V_{s t}$ if $a \neq 1$ and $\alpha \in V_{s t}$ if $a=1$ are also similar to the one above.

Corollary 5.8. Suppose $\sqrt[3]{t s^{-1}} \in k$. Then $T \in$ Aut $\mathscr{J}$, where $T$ is defined by

$$
\left(\begin{array}{ll}
c & \gamma \\
\delta & d
\end{array}\right) T:=\left(\begin{array}{cc}
d & \sqrt[3]{t s^{-1}} \delta \\
\sqrt[3]{s t^{-1} \gamma} & c
\end{array}\right) .
$$

Moreover Aut $\mathcal{J}$ is generated by the $A_{R}$ 's, $E_{\alpha}$ 's, $F_{\beta}$ 's, and $T$.

Proof. That (5.8.1) defines an automorphism of $\mathscr{J}$ is an easy check. The rest follows from Theorem 5.7 and the fact that $T B_{S}=A_{R}$ for all $B_{S} \in$ Aut $\mathscr{J}, S=$ $\left(S_{1}, S_{2}\right)$, where $R=\left(\sqrt[3]{s t^{-1}} S_{1}, \sqrt[3]{t s^{-1}} S_{2}\right)$.

The following relations among the generators of Aut $\mathscr{J}$ are easy verifications.

Proposition 5.9. Let $\theta: G L(\mathscr{S}) \times G L(\mathscr{S}) \rightarrow G L(\mathscr{S}) \times G L(\mathscr{S})$ be defined by $\left(R_{1}, R_{2}\right) \theta:=\left(R_{2}, R_{1}\right)$.

(i) $A_{R} A_{S}=A_{R S}$, where $R S=\left(R_{1} S_{1}, R_{2} S_{2}\right)$ for $R=\left(R_{1}, R_{2}\right)$ and $S=$ $\left(S_{1}, S_{2}\right)$.

(ii) $A_{R} B_{S}=B_{(R \theta) S}$ and $B_{S} A_{R}=B_{S R}$.

(iii) $B_{R} B_{S}=A_{(R \theta) S}$.

(iv) If $\alpha, \beta \in V_{s t}$, then $E_{\alpha} A_{R}=A_{R} E_{\alpha R_{1}}$ and $F_{\beta} A_{R}=A_{R} F_{\beta R_{2}}$ for $R=$ $\left(R_{1}, R_{2}\right)$.

(v) If $\alpha, \beta \in V_{s t}$, then $E_{\alpha} B_{S}=B_{S} F_{\alpha S_{2}}$ and $F_{\beta} B_{S}=B_{S} E_{\beta S_{1}}$ for $S=$ $\left(S_{1}, S_{2}\right)$.

(vi) If $\alpha, \beta \in V_{s t}$, then $E_{\alpha}^{-1}=E_{-\alpha}$ and $F_{\beta}^{-1}=F_{-\beta}$.

(vii) Suppose $\sqrt[3]{t s^{-1}} \in k$. Then $T^{-1}=T$.

Let $G:=\left\{\left(R_{1}, R_{2}\right) \in G L(\mathscr{S}) \times G L(\mathscr{S}) \mid B\left(\gamma R_{i}, \delta R_{j}\right)=B(\gamma, \delta)\right.$ and $(\gamma \delta) R_{i}=\left(\gamma R_{j}\right)\left(\delta R_{j}\right)$ for all $\left.\gamma, \delta \in \mathscr{S}\right\}$ and let $C_{n}$ be the cyclic group of order $n . R=\left(R_{1}, R_{2}\right) \in G$ if and only if $A_{R} \in$ Aut $\mathscr{J}$, so we may regard $G$ as a subgroup of Aut $\mathscr{J}$ via the identification $R \leftrightarrow A_{R}$.

Corollary 5.10. Suppose $\mathscr{J}=\mathscr{J}(\mathscr{S}, B, s, t)$ and $\operatorname{dim} V_{s t}=0$. Then either Aut $\mathscr{J} \cong G$, or $G \triangleleft$ Aut $\mathscr{J}$ and Aut $\mathscr{J} / G \cong C_{2}$. If $\sqrt[3]{s t^{-1}} \in k$, then Aut $\mathscr{J} \cong$ $G \rtimes C_{2}$.

Recall that $\mathscr{G}_{0}:=\left\{\left(D_{1}, D_{2}\right) \in \mathscr{G} \mid D_{1}=D_{2}\right\}$.

Lemma 5.11. Suppose $k$ is algebraically closed, $\operatorname{dim} \mathscr{S}<\infty, \mathscr{S}$ is not abelian, $\operatorname{Der} \mathscr{J}=\mathscr{G}_{0}$, and $\mathscr{S}$ is an irreducible $\mathscr{G}_{0}$-module. Then Aut $\mathscr{J} \cong\left(\operatorname{Aut}_{B} \mathscr{S} \times\right.$ $\left.C_{3}\right) \rtimes C_{2}$, where $\operatorname{Aut}_{B} \mathscr{S}:=\{R \in$ Aut $\mathscr{S} \mid B(\gamma R, \delta R)=B(\gamma, \delta)$ for all $\gamma, \delta \in$ $\mathscr{S}\}$.

Proof. Since $\operatorname{Der} \mathscr{J}=\mathscr{G}_{0}$, where $\mathscr{J}=\mathscr{J}(\mathscr{S}, B, s, t), \operatorname{dim} V_{s t}=0$ by Theorem 2.9. Hence by Corollary 5.10 Aut $\mathscr{J} \cong G \rtimes C$, since $k$ is algebraically closed. Thus it is only necessary to show that $G \cong \operatorname{Aut}_{B} \mathscr{S} \times C_{3}$. Suppose $\left(R_{1}, R_{2}\right) \in G$ and $(D, D) \in \operatorname{Der} \mathcal{J}$. Then $\left(R_{1}, R_{2}\right)^{-1}(D, D)\left(R_{1}, R_{2}\right)=$ $\left(R_{1}^{-1} D R_{1}, R_{2}^{-1} D R_{2}\right) \in \operatorname{Der} \mathcal{J}$. Since $\operatorname{Der} \mathscr{J}=\mathscr{G}_{0}$, we have $R_{1}^{-1} D R_{1}=$ $R_{2}^{-1} D R_{2}$, i.e., $D R_{1} R_{2}^{-1}=R_{1} R_{2}^{-1} D$ for all $(D, D) \in \operatorname{Der} \mathscr{J}$. Since $\mathscr{S}$ is an irreducible $\mathscr{G}_{0}$-module (where the action is defined by $\gamma \cdot(D, D):=\gamma D$ ), we have $R_{1} R_{2}^{-1}=c I$ for some $c \in k, c \neq 0$, by Schur's Lemma. Hence $R_{1}=c R_{2}$. 
Since $\mathscr{S}$ is not abelian and $c(\gamma \delta)=(\gamma \delta) R_{1} R_{2}^{-1}=\left(\gamma R_{2} R_{1}^{-1}\right)\left(\delta R_{2} R_{1}^{-1}\right)=$ $c^{-2}(\gamma \delta)$, we get $c^{3}=1$. Thus $\left(c R_{1}, c\right) \in \operatorname{Aut}_{B} \mathscr{S} \times C_{3}$. Conversely if $(R, c) \in \operatorname{Aut}_{B} \mathscr{S} \times C_{3}$, it is easy to check that $\left(c^{-1} R, c^{-2} R\right) \in G$ and we are done.

We now consider the $\operatorname{dim} V_{s t}=1$ case.

Theorem 5.12. Suppose $k$ is algebraically closed, $\operatorname{dim} \mathscr{S} \geq 4$, and $\operatorname{dim} V_{a}=1$. Then $G \cong H \times k^{*}$, where $H$ is a symplectic group and $k^{*}$ is the multiplicative group of $k$. Also the copy of $H$ in $G$ is a normal subgroup of Aut $\mathscr{J}$.

Proof. By Theorem 4.2, $\mathscr{S}=k \alpha \perp W$ relative to $B$, where $V_{a}=k \alpha$, and the multiplication in $\mathscr{S}$ is given by (4.2.9), where $A \in G L(W)$ is defined by $\gamma A:=\alpha \gamma$. Moreover, for all $\gamma \in W, B(\gamma A, \gamma A)=-a^{-1} B(\alpha, \alpha) B(\gamma, \gamma)$ and $A^{2}=a^{-1} B(\alpha, \alpha) I$. Hence $\langle\gamma, \delta\rangle:=B(\gamma A, \delta)$ is a nondegenerate skewsymmetric bilinear form on $W$ and so $H:=\{R \in G L(W) \mid\langle\gamma R, \delta R\rangle=\langle\gamma, \delta\rangle$ for all $\gamma, \delta \in W\}$ is a symplectic group. For $R \in G L(W)$ define $\bar{R} \in G L(\mathscr{S})$ by $(a \alpha+\gamma) \bar{R}=a \alpha+(\gamma R)$ for $\gamma \in W$. It is easy to check using (4.2.9) that if $R \in H$, then $\left(\bar{R}, \overline{A^{-1} R A}\right) \in G$. Also, if $f \in k^{*}$, then $\left(R_{f}, R_{f^{-1}}\right) \in G$, where $R_{f} \in G L(\mathscr{S})$ is defined by

$$
(a \alpha+\gamma) R_{f}:=a f^{-2} \alpha+f \gamma
$$

for $\gamma \in W$. Thus $H \times k^{*}$ is isomorphic to a subgroup of $G$.

Conversely, suppose $\left(R_{1}, R_{2}\right) \in G$. Then $\alpha R_{i}$ satisfies $(2.5)$ for $i=1,2$ so $\alpha R_{i}=e_{i} \alpha$ for some $e_{i} \in k^{*}, i=1,2$, and since $B\left(\alpha R_{1}, \alpha R_{2}\right)=B(\alpha, \alpha), e_{1}=$ $e_{2}^{-1}=e$. Moreover if $\gamma \in W$ and $\gamma R_{i}=a_{i} \alpha+\delta_{i}$, then $0=B(\alpha, \gamma)=$ $B\left(\alpha R_{j}, \gamma R_{i}\right)=B\left(e_{j} \alpha, a_{i} \alpha+\delta_{i}\right)=e_{j} a_{i} B(\alpha, \alpha)$ implies $a_{i}=0$, i.e., $W R_{i} \subseteq$ $W$. Thus from $(\alpha \gamma) R_{j}=\left(\alpha R_{i}\right)\left(\gamma R_{i}\right)$ we get $\gamma R_{i}=e_{j} \gamma A^{-1} R_{j} A$ for $\gamma \in W$ and from $(\gamma \delta) R_{i}=\left(\gamma R_{j}\right)\left(\delta R_{j}\right)$ we get $e_{i} B(\gamma A, \delta)=B\left(\gamma R_{j} A, \delta R_{j}\right)$ for all $\gamma, \delta \in W$. Let $f \in k^{*}$ such that $e=f^{2}$ and define $S \in G L(W)$ by $S=$ $\left.\left(R_{1} R_{f}\right)\right|_{W}$, where $R_{f}$ is defined by (5.12.1). Then $S \in H$ and $\left(R_{1}, R_{2}\right)=$ $\left(\bar{S}, \overline{A^{-1} S A}\right)\left(R_{f^{-1}}, R_{f}\right)$, so $G \cong H \times k^{*}$. Clearly $H \triangleleft G$. Using the identification of $G$ with a subgroup of Aut $\mathscr{J}$ given above, we can regard $H$ as a subgroup of Aut $\mathcal{J}$. The relations in (iv) of Proposition 5.9 show that $H$ is normalized by the $E_{\alpha}$ 's and $F_{\beta}$ 's. $H$ is also normalized by the mapping $T$ defined by (5.8.1), so $H \triangleleft$ Aut $\mathcal{J}$ by Corollary 5.8.

The rest of the relations in the $\operatorname{dim} V_{s t}=1$ case are given by the following proposition.

Proposition 5.13. Suppose $\operatorname{dim} \mathscr{S} \geq 4, \operatorname{dim} V_{a}=1, \mathscr{J}=\mathscr{J}(\mathscr{S}, B, s, t)$, where st $=a$, and $\alpha, \beta \in V_{a}$. Then

(i) $E_{\alpha} E_{\beta}=E_{\alpha+\beta}$ and $F_{\alpha} F_{\beta}=F_{\alpha+\beta}$.

(ii) If $B(\alpha, \beta)=-1$, then $E_{\alpha} F_{\beta}=B_{S} E_{-\alpha}$, where $S=\left(S_{1}, S_{2}\right)$ is defined by

$$
\begin{aligned}
\delta S_{1} & :=t \beta \delta-B(\alpha, \delta) \alpha, \\
\gamma S_{2} & :=s \alpha \gamma-B(\beta, \gamma) \beta .
\end{aligned}
$$

(iii) If $B(\alpha, \beta) \neq-1$, let $a=-(B(\alpha, \beta)+1), \alpha^{\prime}=a^{-1} \alpha$, and $\beta^{\prime}=a \beta$. Then $E_{\alpha} F_{\beta}=A_{R} F_{-\beta^{\prime}} E_{-\alpha^{\prime}}$, where $R=\left(R_{1}, R_{2}\right)$ is defined by

$$
\gamma R_{1}:=-a \gamma+B(\beta, \gamma)\left[-1+a^{-1}-a^{-2}\right] \alpha,
$$




$$
\delta R_{2}:=-a^{-1} \delta+B(\alpha, \delta)\left[1-a-a^{-1}\right] \beta .
$$

Recall that $C(x, y):=\operatorname{trace}(x y)$ for all $x, y \in \mathcal{J}$. The proof of the following proposition is straightforward.

Proposition 5.14. $C(x E, y E)=C(x, y)$ for all $E \in$ Aut $\mathscr{J}$ and all $x, y \in \mathcal{J}$.

\section{ISOMORPHISMS}

Turning now to isomorphisms between $\mathscr{J}_{1}=\mathscr{J}\left(\mathscr{S}_{1}, B_{1}, s_{1}, t_{1}\right)$ and $\mathscr{J}_{2}=$ $\mathscr{J}\left(\mathscr{S}_{2}, B_{2}, s_{2}, t_{2}\right)$, we have the following theorem, whose proof is quite similar to the proof of Theorem 5.7 and so is omitted.

Theorem 6.1. Suppose $E: \mathscr{J}_{1} \rightarrow \mathscr{J}_{2}$ is an isomorphism of Jordan algebras. Then there is a $C \in$ Aut $\mathscr{J}_{2}$ such that $E C$ has one of the following forms:

(1) $\left(\begin{array}{ll}c & \gamma \\ \delta & d\end{array}\right) E C=\left(\begin{array}{cc}c & \gamma R_{1} \\ \delta R_{2} & d\end{array}\right)$, where $R_{1}, R_{2}: \mathscr{S}_{1} \rightarrow \mathscr{S}_{2}$ satisfy

$$
\begin{aligned}
& B_{2}\left(\gamma R_{1}, \delta R_{2}\right)=B_{1}(\gamma, \delta), \\
& t_{1} t_{2}^{-1}(\gamma \delta) R_{1}=\left(\gamma R_{2}\right)\left(\delta R_{2}\right), \\
& s_{1} s_{2}^{-1}(\gamma \delta) R_{2}=\left(\gamma R_{1}\right)\left(\delta R_{1}\right)
\end{aligned}
$$

for all $\gamma, \delta \in \mathscr{S}_{1}$, or

(2) $\left(\begin{array}{ll}c & \gamma \\ \delta & d\end{array}\right) E C=\left(\begin{array}{cc}d & \delta S_{1} \\ \gamma S_{2} & c\end{array}\right)$ where $S_{1}, S_{2}: \mathscr{S}_{1} \rightarrow \mathscr{S}_{2}$ satisfy

$$
\begin{aligned}
& B_{2}\left(\gamma S_{1}, \delta S_{2}\right)=B_{1}(\gamma, \delta), \\
& s_{1} t_{2}^{-1}(\gamma \delta) S_{1}=\left(\gamma S_{2}\right)\left(\delta S_{2}\right), \\
& t_{1} s_{2}^{-1}(\gamma \delta) S_{2}=\left(\gamma S_{1}\right)\left(\delta S_{1}\right)
\end{aligned}
$$

for all $\gamma, \delta \in \mathscr{S}_{1}$.

Conversely, if $R_{1}, R_{2}: \mathscr{S}_{1} \rightarrow \mathscr{S}_{2}$ satisfy (6.1.1), (6.1.2), and (6.1.3), then $E: \mathscr{J}_{1} \rightarrow \mathscr{J}_{2}$ defined by $\left(\begin{array}{ll}c & \gamma \\ \delta & d\end{array}\right) E:=\left(\begin{array}{cc}c & \gamma R_{1} \\ \delta R_{2} & d\end{array}\right)$ is an isomorphism of Jordan algebras, and if $S_{1}, S_{2}: \mathscr{S}_{1} \rightarrow \mathscr{S}_{2}$ satisfy (6.1.4), (6.1.5), and (6.1.6), then $F: \mathscr{J}_{1} \rightarrow \mathscr{J}_{2}$ defined by $\left(\begin{array}{ll}c & \gamma \\ \delta & d\end{array}\right) F:=\left(\begin{array}{cc}d & \delta S_{1} \\ \gamma S_{2} & c\end{array}\right)$ is an isomorphism of Jordan algebras. Moreover, if $\sqrt[3]{t_{2} s_{2}^{-1}} \in k$ and $E: \mathscr{J}_{1} \rightarrow \mathscr{J}_{2}$ is an isomorphism of Jordan algebras, then it is possible to choose $C \in$ Aut $\mathscr{J}_{2}$ so that $E C$ has form (1).

A bijective linear mapping $R: \mathscr{S}_{1} \rightarrow \mathscr{S}_{2}$ is a scalar isomorphism if there is an $x_{R} \in k, x_{R} \neq 0$, such that for all $\gamma, \delta \in \mathscr{S}_{1}$

$$
(\gamma \delta) R=x_{R}(\gamma R)(\delta R) \text {. }
$$

If there is a scalar isomorphism from $\mathscr{S}_{1}$ to $\mathscr{S}_{2}$, then $\mathscr{S}_{1}$ and $\mathscr{S}_{2}$ are scalar isomorphic. $x_{R}$ is the scaling factor of $R$. Note that $x_{R} R$ is an isomorphism from $\mathscr{S}_{1}$ to $\mathscr{S}_{2}$.

Suppose $S \in G L\left(\mathscr{S}_{1}\right)$ such that for all $\gamma, \delta \in \mathscr{S}_{1}$

$$
B_{1}(\gamma S, \delta)=B_{1}(\gamma, \delta S),
$$

and there is a $w_{S} \in k, w_{S} \neq 0$, such that for all $\gamma, \delta \in \mathscr{S}_{1}$

$$
((\gamma S)(\delta S)) S=w_{S} \gamma \delta
$$


We can define a new product $\cdot s$ on $\mathscr{S}_{1}$ by

$$
\gamma \cdot s \delta:=(\gamma \delta) S
$$

and a new nondegenerate symmetric bilinear form $B_{S}$ by

$$
B_{S}(\gamma, \delta)=B_{1}\left(\gamma S^{-1}, \delta\right) .
$$

It is easy to check that $\mathscr{S}_{1}$ with the product $\cdot s$ is an anticommutative algebra and $B_{S}$ is associative. We will refer to $\mathscr{S}_{1}$ with the product ${ }_{s}$ as $\mathscr{S}_{S}$.

Theorem 6.7. Suppose $\sqrt[3]{t_{2} s_{2}^{-1}} \in k$. Then $\mathscr{J}_{1} \cong \mathscr{J}_{2}$ as Jordan algebras iff there is an $S \in G L\left(\mathscr{S}_{1}\right)$ satisfying (6.3) and (6.4) with $w_{S}=t_{1}^{-1} s_{1} t_{2} s_{2}^{-1}$ and a scalar isomorphism $R: \mathscr{S}_{S} \rightarrow \mathscr{I}_{2}$ with $x_{R}=t_{1}^{-1} t_{2}$ such that $R$ is an isometry from $B_{S}$ to $B_{2}$.

Proof. First suppose $S \in G L\left(\mathscr{S}_{1}\right)$ satisfies (6.3) and (6.4) with $w_{S}=t_{1}^{-1} s_{1} t_{2} s_{2}^{-1}$, and $R: \mathscr{S}_{S} \rightarrow \mathscr{S}_{2}$ is a scalar isomorphism with $x_{R}=t_{1}^{-1} t_{2}$ such that $R$ is an isometry from $B_{S}$ to $B_{2}$. Define $E: \mathscr{J}_{1} \rightarrow \mathscr{F}_{2}$ by $\left(\begin{array}{cc}c & \gamma \\ \delta & d\end{array}\right) E:=\left(\begin{array}{cc}c & \gamma S R \\ \delta R & d\end{array}\right)$. Then $E$ is an isomorphism of Jordan algebras.

Conversely, suppose $\mathscr{F}_{1} \cong \mathscr{Z}_{2}$. By Theorem 6.1 we can find an isomorphism $E: \mathscr{Z}_{1} \rightarrow \mathscr{J}_{2}$ of Jordan algebras such that $\left(\begin{array}{ll}c & \gamma \\ \delta & d\end{array}\right) E=\left(\begin{array}{cc}c & \gamma R_{1} \\ \delta R_{2} & d\end{array}\right)$, where $R_{1}, R_{2}$ : $\mathscr{S}_{1} \rightarrow \mathscr{S}_{2}$ satisfy (6.1.1), (6.1.2), and (6.1.3). If we let $S=R_{1} R_{2}^{-1}$ and $R=R_{2}$, then $S$ satisfies (6.3) and (6.4) with $w_{S}=t_{1}^{-1} s_{1} t_{2} s_{2}^{-1}, R: \mathscr{S}_{S} \rightarrow \mathscr{S}_{2}$ is a scalar isomorphism with $x_{R}=t_{1}^{-1} t_{2}$, and $R$ is an isometry from $B_{S}$ to $B_{2}$.

Suppose $\mathscr{S}_{1}, B_{1}, s_{1}, t_{1}$ are given and $S \in G L\left(\mathscr{S}_{1}\right)$ satisfies (6.3) and (6.4) for some $w_{S} \in k, w_{S} \neq 0$. For any $T \in G L\left(\mathscr{S}_{1}\right)$ and any $x \in k, x \neq 0$, we can define a new product ${ }^{T} T$ and a new bilinear form $B_{T}$ on $\mathscr{S}_{1}$ by $\gamma \cdot T \delta:=\left[\left(\gamma T^{-1}\right) \cdot s\left(\delta T^{-1}\right)\right] T$, where $\cdot S$ is defined by $(6.5)$, and $B_{T}(\gamma, \delta):=$ $x^{2} B_{S}\left(\gamma T^{-1}, \delta T^{-1}\right)$, where $B_{S}$ is defined by (6.6). Now if we define $R:=$ $x^{-1} T$, then $R$ is an isometric scalar isomorphism from $\left(\mathscr{S}_{1}, \cdot_{S}, B_{S}\right)$ to $\left(\mathscr{S}_{1}, \cdot_{T}, B_{T}\right)$ and $x_{R}=x$. By Theorem 6.7

$$
\mathscr{J}\left(\mathscr{S}_{1}, B_{1}, s_{1}, t_{1}\right) \cong \mathscr{J}\left(\mathscr{S}_{1}, \cdot_{T}, B_{T}, s_{2}, t_{2}\right),
$$

where $t_{2}=x t_{1}$ and $s_{2}=t_{1}^{-1} s_{1} t_{2} w_{S}^{-1}$ and every $\left(\mathscr{S}_{2}, B_{2}\right)$ such that $\mathscr{J}_{1} \cong \mathscr{J}_{2}$ is isometrically isomorphic to $\left(\mathscr{S}_{1}, \cdot_{T}, B_{T}\right)$ for some choice of $S, T$, and $x$. Thus it is clear that the question of which $\left(\mathscr{S}_{2}, B_{2}\right)$ gives $\mathscr{L}_{2}$ isomorphic to $\mathscr{J}_{1}$ for given $\mathscr{S}_{1}, B_{1}, s_{1}, t_{1}$ reduces to the study of those $S \in G L\left(\mathscr{S}_{1}\right)$ which satisfy (6.3) and (6.4).

Finally we have the following proposition, whose proof is straightforward.

Proposition 6.8. Suppose $S, T \in G L\left(\mathscr{S}_{1}\right)$ both satisfy (6.3) and (6.4) with $w_{S}=$ $w_{T}$. Then $\mathscr{S}_{S} \cong \mathscr{S}_{T}$ under an isometry from $B_{S}$ to $B_{T}$ iff there are $R_{1}, R_{2} \in$ $G L\left(\mathscr{S}_{1}\right)$ such that for all $\gamma, \delta \in \mathscr{S}_{1}$

$$
B_{1}\left(\gamma R_{1}, \delta R_{2}\right)=B_{1}(\gamma, \delta)
$$

and

$$
(\gamma \delta) R_{i}=\left(\gamma R_{j}\right)\left(\delta R_{j}\right) \text { for } i, j=1,2, i \neq j
$$


and $T=R_{1} S R_{2}^{-1}$. In this case, $R_{2}: \mathscr{S}_{T} \rightarrow \mathscr{S}_{S}$ is an isometric isomorphism. Also, if $S \in G L\left(\mathscr{S}_{1}\right)$ satisfies (6.3) and (6.4) and $R_{1}, R_{2} \in G L\left(\mathscr{S}_{1}\right)$ satisfy (6.8.1) and (6.8.2), then $T:=R_{1} S R_{2}^{-1}$ satisfies (6.3) and (6.4), with $w_{T}=w_{S}$.

Note that if $R_{1}, R_{2} \in G L\left(\mathscr{S}_{1}\right)$ satisfy (6.8.1) and (6.8.2), then $A_{R} \in$ Aut $\mathscr{L}_{1}$ for $R=\left(R_{1}, R_{2}\right)$. The actual computation of $\left\{S \in G L\left(\mathscr{S}_{1}\right) \mid S\right.$ satisfies (6.3) and (6.4) $\}$ may be quite difficult for particular $\mathscr{S}_{1}$, but we note that (6.3) and (6.4) together imply that $S$ is in the structure group of $\mathscr{S}_{1}$, about which much is known, at least for commutative Jordan algebras.

\section{ReLAXING THE $s t \neq 0$ REQUIREMENT}

If we assume that $s$ or $t$ is zero, then results similar to the ones in the previous sections are true and are stated here without proof. Verification of these statements depends on calculations that are like those appearing elsewhere in this paper.

Theorem 7.1. Suppose $s=0$ or $t=0$ and $\mathscr{J}=\mathscr{J}(\mathscr{S}, B, s, t)$. Then

(i) $\mathcal{J}$ is central simple.

(ii) Let $\mathscr{G}$ be the set of $\left(D_{1}, D_{2}\right) \in \operatorname{End}_{k} \mathscr{S} \oplus \operatorname{End}_{k} \mathscr{S}$ satisfying the following for all $\gamma, \delta \in \mathscr{S}$ :

$$
B\left(\gamma D_{i}, \delta\right)=-B\left(\gamma, \delta D_{j}\right) \text { for } i, j=1,2, i \neq j,
$$

and

$$
\text { if } s \neq 0:(\gamma \delta) D_{2}=\left(\gamma D_{1}\right) \delta+\gamma\left(\delta D_{1}\right)
$$

or

$$
\text { if } t \neq 0:(\gamma \delta) D_{1}=\left(\gamma D_{2}\right) \delta+\gamma\left(\delta D_{2}\right) \text {. }
$$

Then $\operatorname{Der} \mathscr{J}=\mathscr{G}$ where the action of $\left(D_{1}, D_{2}\right) \in \mathscr{G}$ is given by $\left(\begin{array}{ll}c & \gamma \\ \delta & d\end{array}\right)\left(D_{1}, D_{2}\right)$ $:=\left(\begin{array}{cc}0 & \gamma D_{1} \\ \delta D_{2} & 0\end{array}\right)$.

(iii) Let $R=\left(R_{1}, R_{2}\right) \in G L(\mathscr{S}) \times G L(\mathscr{S})$ satisfy

$$
B\left(\gamma R_{1}, \delta R_{2}\right)=B(\gamma, \delta) \text { for all } \gamma, \delta \in \mathscr{S} \text {. }
$$

Define $A_{R}, B_{R} \in G L(\mathcal{J})$ by

$$
\left(\begin{array}{ll}
c & \gamma \\
\delta & d
\end{array}\right) A_{R}:=\left(\begin{array}{cc}
c & \gamma R_{1} \\
\delta R_{2} & d
\end{array}\right) \text { and }\left(\begin{array}{ll}
c & \gamma \\
\delta & d
\end{array}\right) B_{R}:=\left(\begin{array}{cc}
d & \delta R_{1} \\
\gamma R_{2} & c
\end{array}\right) \text {. }
$$

Then:

(1) If $s=t=0$ or $\mathscr{S}$ is abelian, Aut $\mathscr{J}=\left\{A_{R}, B_{R} \mid R\right.$ satisfies (7.1.4) $\}$.

(2) If $s \neq 0, t=0$, and $\mathscr{S}$ is not abelian, Aut $\mathscr{J}=\left\{A_{R} \mid R\right.$ satisfies (7.1.4) and $(\gamma \delta) R_{2}=\left(\gamma R_{1}\right)\left(\delta R_{1}\right)$ for all $\left.\gamma, \delta \in \mathscr{S}\right\}$.

(3) If $s=0, t \neq 0$, and $\mathscr{S}$ is not abelian, Aut $\mathscr{J}=\left\{A_{R} \mid R\right.$ satisfies (7.1.4) and $(\gamma \delta) R_{1}=\left(\gamma R_{2}\right)\left(\delta R_{2}\right)$ for all $\left.\gamma, \delta \in \mathscr{S}\right\}$.

Finally, we note that results similar to those of Theorem 7.1 are true for $\mathcal{J}$ when the multiplication in $\mathscr{J}$ is not defined by (1.2), but rather by the more general formula

$$
\begin{aligned}
& \left(\begin{array}{ll}
a & \alpha \\
\beta & b
\end{array}\right)\left(\begin{array}{ll}
c & \gamma \\
\delta & d
\end{array}\right) \\
& \quad:=\left(\begin{array}{cc}
a c+z_{1} B(\alpha, \delta) & x_{1} a \gamma+\left(1-x_{1}\right) b \gamma+y_{2} d \alpha \\
x_{2} b \delta+\left(1-x_{2}\right) a \delta+y_{1} c \beta & +\left(1-y_{2}\right) c \alpha+t \beta \delta \\
+\left(1-y_{1}\right) d \beta+s \alpha \gamma & b d+z_{2} B(\beta, \gamma)
\end{array}\right)
\end{aligned}
$$


where $x_{1}, x_{2}, y_{1}, y_{2}, z_{1}, z_{2} \in k$ are arbitrary with $z_{1} \neq 0, z_{2} \neq 0$. We get (1.2) from (7.2) by choosing $x_{1}=x_{2}=y_{1}=y_{2}=z_{1}=z_{2}=1$. In fact, the only choices for these scalars that give a noncommutative Jordan algebra are $x_{1}=x_{2}=y_{1}=y_{2}=1$ and $z_{1}=z_{2}$ and we have dealt with these in our previous results.

\section{REFERENCES}

1. J. E. Humphreys, Introduction to Lie algebras and representation theory, 2nd printing, revised, Springer-Verlag, New York, 1972.

2. N. Jacobson, Lie algebras, Dover, New York, 1979.

3. __ Some groups of transformations defined by Jordan algebras. I, J. Reine Angew. Math. 201 (1959), 178-195.

4. L. J. Paige, A note on noncommutative Jordan algebras, Portugal. Math. 16 (1957), 15-18.

5. A. A. Sagle, On anti-commutative algebras with an invariant form, Canad. J. Math. 16 (1964), 370-378.

6. G. B. Seligman, Modular Lie algebras, Springer-Verlag, New York, 1967.

Department of Mathematics, The Ohio State University, Columbus, Ohio 43210

Department of Mathematics, Indiana State University, Terre Haute, Indiana 47809 